\title{
A SIGNIFICANT POPULATION OF CANDIDATE NEW MEMBERS OF THE $\rho$ OPHIUCHI CLUSTER
}

\author{
Mary Barsony ${ }^{1,5,6}$, Karl E. Haisch JR. ${ }^{2,5}$, Kenneth A. Marsh ${ }^{3}$, and Chris McCarthy ${ }^{4}$ \\ ${ }^{1}$ SETI Institute, 189 Bernardo Ave., Suite 100, Mt. View, CA 94043, USA; mbarsony@ seti.org \\ ${ }^{2}$ Physics Department, Utah Valley University, 800 W. University Pkwy., Orem, UT 85058-5999, USA; Karl.Haisch@uvu.edu \\ ${ }^{3}$ Infrared Processing and Analysis Center, California Institute of Technology 100-22, Pasadena, CA 91125, USA; kam@ipac.caltech.edu \\ ${ }^{4}$ Department of Physics \& Astronomy, San Francisco State University, 1600 Holloway Ave., San Francisco, CA 94132, USA; exoplanet@ gmail.com \\ Received 2011 December 14; accepted 2012 February 17; published 2012 April 30
}

\begin{abstract}
We present a general method for identifying the pre-main-sequence population of any star-forming region, unbiased with respect to the presence or absence of disks, in contrast to samples selected primarily via their mid-infrared emission from Spitzer surveys. We have applied this technique to a new, deep, wide-field, near-infrared imaging survey of the $\rho$ Ophiuchi cloud core to search for candidate low-mass members. In conjunction with published Spitzer IRAC photometry and least-squares fits of model spectra (COND, DUSTY, NextGen, and blackbody) to the observed spectral energy distributions, we have identified 948 candidate cloud members within our $90 \%$ completeness limits of $J=20.0, H=20.0$, and $K_{s}=18.50$. This population represents a factor of $\sim 3$ increase in the number of known young stellar objects in the $\rho$ Ophiuchi cloud. A large fraction of the candidate cluster members $(81 \% \pm 3 \%)$ exhibit infrared excess emission consistent with the presence of disks, thus strengthening the possibility of their being bona fide cloud members. Spectroscopic follow-up will confirm the nature of individual objects, better constrain their parameters, and allow an initial mass function to be derived.
\end{abstract}

Key words: infrared: stars - ISM: individual objects (Rho Ophiuchi) - stars: pre-main sequence

Online-only material: machine-readable table

\section{INTRODUCTION}

Substellar objects, including brown dwarfs, sub-brown dwarfs, and free-floating objects of planetary mass, are all at their most luminous (by orders of magnitude) upon formation. Therefore, the nearest and youngest star-forming regions (SFRs) present the best opportunity to determine the shape of the initial mass function (IMF) at the lowest masses. Theories of star formation that attempt to predict IMFs (Kroupa 2011; Hennebelle \& Chabrier 2011; McKee \& Ostriker 2007) require observational constraints, especially at the low-mass end. Specifically, one pressing open question is, What is the lowest mass object that can form via the usual star formation process?

The advent of large-format near-infrared (NIR) array detectors has opened the door to surveying large angular extents of nearby SFRs with sensitivity sufficient to uncover planetary mass objects (PMOs). The potential now exists to determine the IMF shapes of the nearest and youngest SFRs through the substellar regime down to $\sim 1-2 M_{\text {Jup }}$.

Recent work on some nearby SFRs has determined IMFs in a statistical sense only, by deriving luminosity functions (LFs), deduced from imaging data obtained in just a single NIR waveband. For example, IMFs were derived from observed $K$-band luminosity functions (KLFs) for the Orion Nebula Cluster (ONC; Muench et al. 2002) and IC 348 (Muench et al. 2003) or from the observed $J$-band luminosity function (JLF) for IC 348 (Preibisch et al. 2003). This method for IMF determination relies on (1) an assumed form of the mass function, (2) an assumed age distribution, and (3) premain-sequence (PMS) model tracks that give the single filter brightness for a given mass. Additional complications that arise

\footnotetext{
5 Visiting Astronomer at the Anglo-Australian Telescope, Siding Spring, Australia.

6 Department of Physics and Astronomy, San Francisco State University, 1600 Holloway Ave., San Francisco, CA 94132, USA.
}

for this (and other) method(s) of IMF determination include distinguishing cluster members from non-members, accounting for the extinction to each object, and correcting for excess emission due to circumstellar material/disks to the observed KLFs (the latter effect is minimized for JLFs).

A summary of published IMFs that make use of NIR photometric data for six nearby SFRs is presented in Table 1. Distances, ages, telescope/instrument combinations used, completeness limits reached, and references are listed. The potential for deriving an IMF in $\rho$ Oph is shown by listing the parameters of our survey in the last entry of Table 1 .

Alternative approaches for IMF determinations of SFRs, applicable to low-extinction regions, include converting the observed JLF to an LF with the aid of model PMS tracks by applying bolometric corrections (BCs) derived from field stars. PMS tracks, for a presumed cluster age, are then used to convert the LF to a mass function (e.g., Lodieu et al. 2009 for $\sigma$ Ori). This approach is aided by complementary spectroscopy, since the BCs are a function of spectral type (e.g., Caballero et al. 2007). Optical multi-object spectroscopy has been used for IMF determinations in relatively low extinction regions of SFRs to derive cluster membership, extinction, and spectral types for individual candidate young stellar objects (YSOs). Broadband photometry is then used, in conjunction with $\mathrm{BCs}$, to derive individual YSO luminosities. Each YSO is plotted on an H-R diagram, and comparison with a set of PMS tracks is used to derive a mass and age for each cluster member (e.g., Luhman 2004 for Taurus; Luhman 2007 for Cha I).

Finally, fitting of multi-wavelength spectral energy distributions (SEDs) has been used to derive dereddened effective temperatures $\left(T_{\text {eff }}\right)$ for cloud members. PMS tracks, for an assumed cluster age, are then used to derive mass values for a given $T_{\text {eff }}$ (e.g., Marsh et al. 2010b for $\rho \mathrm{Oph}$ ). This approach, however, is problematic for treating YSOs with circumstellar disks and/or envelopes. 
Table 1

Nearby SFRs with Published IMFs Using NIR Imaging Data

\begin{tabular}{|c|c|c|c|c|c|c|c|c|}
\hline SFR & $\begin{array}{l}\text { Distance } \\
\quad(\mathrm{pc})\end{array}$ & $\begin{array}{l}\text { Age } \\
(\mathrm{Myr})\end{array}$ & $\begin{array}{l}\text { Telescope } \\
\text { IR Camera }\end{array}$ & FOV & $\begin{array}{c}J \\
(\mathrm{mag})\end{array}$ & $\begin{array}{c}H \\
(\mathrm{mag})\end{array}$ & $\begin{array}{c}K_{s} \\
(\mathrm{mag})\end{array}$ & Ref \\
\hline \multirow[t]{2}{*}{ IC 348} & 300 & 2 & $\begin{array}{l}\text { KPNO } 2.1 \mathrm{~m} \\
\text { FLAMINGOS }\end{array}$ & $20.5 \times 20^{\prime} .5$ & 18.82 & 18.04 & 17.72 & (1) \\
\hline & & & $\begin{array}{l}\text { Calar-Alto } 3.5 \mathrm{~m} \\
\text { Omega Prime }\end{array}$ & $18^{\prime} \cdot 6 \times 18^{\prime} \cdot 4$ & 19.5 &.$^{\mathrm{a}}$ &.$^{\mathrm{a}}$ & (2) \\
\hline ONC & 430 & $0.8 \pm 0.6$ & $\begin{array}{c}\text { NTT } 3.5 \mathrm{~m} / \mathrm{SOFI}+ \\
\text { FLWO } 1.2 \mathrm{~m} / \mathrm{STELIRcam}\end{array}$ & $5^{\prime} \times 5^{\prime}$ & 18.15 & 18.7 & 17.5 & (3) \\
\hline Cha I & $160-170$ & $\sim 2$ & $\begin{array}{c}\text { 2MASS + } \\
\text { CTIO } 4 \text { m/ISPI }\end{array}$ & $\begin{array}{c}1.5 \times 0.35 \\
13.2 \times 16^{\prime} .8\end{array}$ & $\begin{array}{c}15.75 \\
18.5\end{array}$ & $\begin{array}{l}15.25 \\
18.25\end{array}$ & $\begin{array}{c}14.3^{\mathrm{b}} \\
\ldots\end{array}$ & (4) \\
\hline Taurus & 140 & $\begin{array}{c}1-1.5^{\mathrm{d}} \\
3^{\mathrm{e}}\end{array}$ & $\begin{array}{l}\text { 2MASS } \\
\text { 2MASS }\end{array}$ & $\begin{array}{l}2.84 \mathrm{deg}^{2} \\
1.32 \mathrm{deg}^{2}\end{array}$ & 15.75 & 15.25 & $14.3^{\mathrm{a}}$ & (5) \\
\hline \multirow[t]{2}{*}{$\sigma$ Ori } & $360_{-60}^{+70}$ & $3 \pm 2$ & $\begin{array}{l}\text { VLT } 8.2 \mathrm{~m} \\
\text { ISAAC }\end{array}$ & $790 \operatorname{arcmin}^{2}$ & 20.6 & $\ldots^{a}$ & $\ldots^{\mathrm{a}}$ & (6) \\
\hline & & & $\begin{array}{l}\text { UKIRT } 3.8 \mathrm{~m} \\
\text { WFCAM }\end{array}$ & $0.78 \mathrm{deg}^{2}$ & 19.0 & 18.4 & 18.0 & (7) \\
\hline$\rho \mathrm{Oph}$ & 124 & 1 & 2MASS & $1^{\circ} \times 9.3$ & 20.5 & 20.0 & 19.0 & (8) \\
\hline$\rho \mathrm{Oph}$ & 124 & 1 & $\begin{array}{l}\text { AAO- } 3.8 \mathrm{~m} \\
\text { IRIS2 }\end{array}$ & $920 \operatorname{arcmin}^{2}$ & 20.0 & 20.0 & 18.5 & (9) \\
\hline
\end{tabular}

Notes.

References. (1) Muench et al. 2003; (2) Preibisch et al. 2003; (3) Muench et al. 2002; (4) Luhman 2007; (5) Luhman 2004; (6)

Caballero et al. 2007; (7) Lodieu et al. 2009; (8) Marsh et al. 2010b; (9) this work.

${ }^{\text {a }}$ No data acquired at this wavelength.

b 2MASS $K_{s}$ completeness from http://www.ipac.caltech.edu/2mass/releases/allsky/doc/sec2_2b.html.

${ }^{\mathrm{c}}$ Data acquired, but $K_{s}$ completeness limit not published.

${ }^{\mathrm{d}}$ For field centered at $\mathrm{J} 2000 \alpha=4^{\mathrm{h}} 39^{\mathrm{m}} 00^{\mathrm{s}}, \delta=25^{\circ} 46^{\prime} 00^{\prime \prime}$.

${ }^{\mathrm{e}}$ For field centered at $\mathrm{J} 2000 \alpha=4^{\mathrm{h}} 55^{\mathrm{m}} 00^{\mathrm{s}}, \delta=30^{\circ} 24^{\prime} 30^{\prime \prime}$.

Table 2

Substellar Objects in Nearby SFRs

\begin{tabular}{lcccc}
\hline \hline SFR & $\begin{array}{c}\text { B.D./Star } \\
\text { Ratio }\end{array}$ & Ref. & $\begin{array}{c}\text { No. of } \\
\text { Candidate } \\
\text { PMOs }\end{array}$ & Ref. \\
\hline IC 348 & $15 \%-25 \%$ & $(1,2)$ & 1 & $(3)$ \\
ONC & $30 \%$ & $(4,5)$ & 10 & $(6)$ \\
NGC 1333 & $54 \%$ & $(7)$ & $142-421$ & $(7)$ \\
Cha I & $67 \%$ & $(8)$ & 0 & $(8)$ \\
Taurus & $17 \%$ & $(9)$ & 10 & $(9)$ \\
$\sigma$ Ori & $17 \%$ & $(10)$ & 0 & $(10)$ \\
Lupus & $23 \%$ & $(11)$ & 17 & $(12)$ \\
$\rho$ Oph & $\ldots \mathrm{c}^{\mathrm{c}}$ & $(13)$ & 0 & $(13)$ \\
\hline
\end{tabular}

Notes.

References. (1) Muench et al. 2003; (2) Luhman et al. 2003; (3) Burgess et al. 2009; (4) Slesnick et al. 2004; (5) Andersen et al. 2008; (6) Weights et al. 2009; (7) Robberto et al. 2010; (8) Scholz et al. 2009; (9) Mužić et al. 2011; (10) Luhman (2004); (11) Lodieu et al. 2009; (12) Figure 2 of Bihain et al. 2009; (13) Comerón 2011; (14) Alves de Oliveira et al. 2010; (15) Wilking et al. 2008; (16) Marsh et al. 2010a; (17) Haisch et al. 2010.

${ }^{a}$ Only isolated objects are listed here, thus leaving out TMR-1c in Taurus (Riaz \& Martín 2011), the candidate PMO companion to 2M J044144 (Todorov et al. 2010), and the candidate PMO companion to Par-Lup3-4 (Comerón 2011).

${ }^{\mathrm{b}}$ For $d=352 \mathrm{pc} ; 14 \%$ for $d=440 \mathrm{pc}$.

c Although no substellar objects were found in the survey of (13), no definitive value for this ratio can be derived, due to small number statistics.

${ }^{\mathrm{d}}$ Approximation using 110 candidate substellar members (14) and 316 members (15).

Related to the determination of the shape of the IMF at the lowest masses is the search for a low-mass cutoff of the IMF. This search has been the focus of many recent investigations. We list those making use of deep, NIR imaging in Table 2. In the IC 348 cluster, one new candidate PMO has been proposed but awaits spectroscopic confirmation (Burgess et al. 2009). The most recent deep, large-area, NIR study of the ONC covered a $30^{\prime} \times 40^{\prime}$ region to $J \simeq 19.5 \mathrm{mag}, H \simeq 18.0 \mathrm{mag}$, and $K_{s} \simeq 18.5 \mathrm{mag}(3 \sigma)$, a sensitivity sufficient to detect $1 \mathrm{Myr}$ old PMOs to $A_{V} \simeq 10 \mathrm{mag}$ of extinction (Robberto et al. 2010). These authors found 1298 sources in the reddened brown dwarf region and 142 in the reddened PMO region of the $H$ versus $J-H$ color-magnitude diagram. The source distribution in the corresponding regions of the $K_{s}$ versus $H-K_{s}$ color-magnitude diagram yielded 2134 sources in the reddened brown dwarf region and 421 sources in the reddened PMO region. In NGC 1333, no PMOs have been found, but 19 spectroscopically confirmed brown dwarfs have, from a sample of 36 objects with $i^{\prime}-z^{\prime}$ colors expected for young, very low mass objects (Scholz et al. 2009). This, despite the sensitivity of the survey to mass limits of $0.008 M_{\odot}$ for $A_{V} \leqslant 10 \mathrm{mag}$ and $0.004 M_{\odot}$ for $A_{V} \leqslant 5 \mathrm{mag}$, led the authors to the conjecture that the low-mass cutoff corresponding to $T_{\text {eff }}<2500 \mathrm{~K}$ has been found for this cluster. In the Cha I SFR, a deep optical imaging survey, with follow-up deep NIR photometry, sensitive to mass limits of $0.003-0.005 M_{\odot}$ for $A_{V} \leqslant 5$, with follow-up low-resolution optical spectroscopy (Mužić et al. 2011), found no new confirmed substellar objects, beyond those found in Luhman (2007). The more recent study placed upper limits on the number of missing planetary mass members (down to $\left.\sim 0.008 M_{\odot}\right)$ of $\leqslant 3 \%(\leqslant 7)$ of the currently known YSO population in Chamaeleon I. Due to its large angular extent, searches for PMOs in the Taurus SFR have been targeted toward known members, be they YSOs or young brown dwarfs. Two notable PMO candidates discovered in this way, necessarily members of multiple systems, are TMR-1c (Terebey et al. 1998; Riaz \& Martín 2011) and 2MASS J04414489+2301513 (Todorov et al. 2010). Large-area surveys of Taurus using Two Micron All-Sky Survey (2MASS) and Spitzer data have 
been used to search for new members, including brown dwarfs (Luhman et al. 2006, 2009a, 2009b; Rebull et al. 2010), bringing the total list of known Taurus members to 318 (Luhman et al. 2010), of which 43 are spectroscopically confirmed as substellar (Monin et al. 2010). In the $\sigma$ Ori cluster, a careful re-analysis of archival data, supplemented by newer large-area, sensitive imaging, led to the discovery of three new planetary mass candidates down to $\sim 4 M_{\text {Jup }}$, bringing to 17 the total number of candidate PMOs in this region (Bihain et al. 2009). In the recent deep NIR imaging study of Lupus III, no PMOs (corresponding to late $\mathrm{T}$ spectral types) but 17 substellar candidates (with 1700 $\mathrm{K} \leqslant T_{\text {eff }} \leqslant 3000 \mathrm{~K}$ ) have been identified (Comerón 2011).

The subject of this study, the $\rho$ Oph SFR, is an especially attractive target, due to its relatively compact area, proximity ( $d=120 \pm 5$ pc; Loinard et al. 2008), richness of its embedded young stellar population (Wilking et al. 2008; Barsony et al. 2005), and youth 1 Myr (Luhman \& Rieke 1999; Prato et al. 2003; Wilking et al. 2005). As such, it has been the target of several, deep, large-area NIR surveys whose stated aim is finding its lowest mass members. In their sensitive NIR survey of $\sim 1 \mathrm{deg}^{2}$ of the main $\rho$ Ophiuchi cloud core, Alves de Oliveira et al. (2010) report the detection of $\sim 5.7 \times 10^{4}$ objects, which they winnowed down using various color-magnitude diagrams guided by the 1 Myr DUSTY isochrone of Chabrier et al. (2000). Their final list of 110 candidate substellar members includes 80 that are newly identified. Another recent survey searching for the lowest mass member in $\rho$ Oph covered a $31^{\prime} .5 \times 26^{\prime}$ area in the $i J K_{s}$ filters. This investigation resulted in the discovery of one new, spectroscopically confirmed, brown dwarf (Geers et al. 2011). Furthermore, these same authors identified 27 brown dwarf candidates (11 of which have previous spectroscopic confirmation) using Spitzer photometry. Analysis of the 2MASS calibration strip, running along a $9^{\prime}$ wide swath through the $\rho$ Oph core, resulted in the identification of 11 possible PMOs ( $T \leqslant 1800 \mathrm{~K}$ ) of $\sim 115$ cluster-member candidates (Marsh et al. $2010 b$ ). Follow-up spectroscopy of seven of these resulted in the discovery of a single PMO (Marsh et al. 2010a).

An alternate approach that has been used to search for the lowest mass member of the $\rho \mathrm{Oph}$ core is to target the spectroscopic signature of methane $\left(\mathrm{CH}_{4}\right)$ absorption, found in lowtemperature $\left(T_{\text {eff }} \leqslant 1500 \mathrm{~K}\right)$ atmospheres. Images are acquired through specially designed adjacent narrowband methane-off $(\sim 1.6 \mu \mathrm{m})$ and methane-on $(\sim 1.7 \mu \mathrm{m})$ filters covering the $H$ band. Methane-absorbing objects, which would have masses of $\sim 1-2 M_{\text {Jup }}$ at the age and distance of the $\rho$ Oph core, would appear uniquely and characteristically bright in methane-off minus methane-on differential images. This technique has been used to survey a $920 \mathrm{arcmin}^{2}$ region of the $\rho$ Oph core to identify 22 planetary mass candidates (Haisch et al. 2010).

Spectroscopic follow-up of these candidates is currently being carried out by our team. As a part of our ongoing $H$-band methane-filter imaging program of nearby SFRs to discover their lowest mass members, we are also acquiring complementary deep $J$ and $K_{s}$ data. In this paper, we report the discovery of 948 candidate low-mass members from combined, deep, $J H K_{S}$ imaging of the central $920 \mathrm{arcmin}^{2}$ area of the $\rho$ Ophiuchi cloud core, supplemented by Spitzer data.

The new observations and data reduction methods are described in Section 2. Results using modeling of our deep $J H K_{S}$ photometry combined with Spitzer photometry are described in Section 3. Properties of the newly discovered sources, estimation of sample contamination, and detailed comparisons with recently published deep, large-area NIR imaging surveys are contained in Section 4. The summary and conclusions are presented in Section 5.

\section{OBSERVATIONS AND DATA REDUCTION}

Observations of the $\rho$ Ophiuchi cloud core were obtained during the period 2008 May 23-26 with the IRIS2 NIR imager/spectrograph on the Australian Astronomical Observatory's 4 m telescope (AAT). IRIS2 consists of a Hawaii $\mathrm{HgCdTe}$ $1024 \times 1024$ array that, when mounted at the $f / 8$ Cassegrain focus on the AAT, yields a plate scale of 0.45 pixel $^{-1}$ with a corresponding field of view of approximately 7 '.7 $\times$ 7'.7. For all observations, the $J, K_{s}(1.25,2.14 \mu \mathrm{m}), \mathrm{CH}_{4 s}(1.59 \mu \mathrm{m})$, and $\mathrm{CH}_{4 l}(1.673 \mu \mathrm{m})$ filters were used. Details of our observation and data reduction procedures for all filters are discussed in Haisch et al. (2010). However, because this paper makes extensive use of the $J$ and $K_{s}$ data, which the previous work did not, we summarize below the observations and image reduction process for these filters.

Nineteen IRIS2 fields, centered at $\alpha=16^{\mathrm{h}} 26^{\mathrm{m}} 56^{\mathrm{s}} .34, \delta=$ $-24^{\circ} 28^{\prime} 52^{\prime \prime} .22$ (J2000), were observed in a rectangular pattern covering an area of $\sim 920 \mathrm{arcmin}^{2}$ on the sky. The observed area is shown by the solid outlines in Figure 1, superposed on the extinction map that was derived from the 2MASS catalog ${ }^{7}$ as part of the COMPLETE project (Ridge et al. 2006; Lombardi et al. 2008) using the NICER algorithm (Lombardi \& Alves 2001). Each field was spatially overlapped by 1 arcmin in both right ascension and declination to allow for redundancy of photometric measurements of sources located in the overlapped regions. All fields were observed in the Mauna Kea Observatory (MKO) photometric system $J$ and $K_{s}$ filters in a five-point dither pattern with $30^{\prime \prime}$ offsets between each dither. Integration times at each dither position for the $J$ and $K_{s}$ filters were $15 \mathrm{~s} \times 4$ coadds and $6 \mathrm{~s} \times 10 \mathrm{co}$-adds, respectively, for a total integration time of 5 minutes in each filter. The FWHM for all observations varied between approximately 2.2 and 3.1 pixels $\left(\sim 1^{\prime \prime} .0-1^{\prime \prime} .4\right)$, with the worst seeing being at the shortest wavelengths (e.g., the $J$ filter).

All data were reduced using the Image Reduction and Analysis Facility (IRAF). ${ }^{8}$ An average dark frame was constructed from the dark frames taken at the beginning and end of each night's observations. This dark frame was subtracted from all target observations to yield dark subtracted images. Sky frames in each filter were individually made for each observation by median-combining all five $J$ - and $K_{s}$-band frames for each field. The individual sky frames were normalized to produce flat fields for each target frame. All target frames were processed by subtracting the appropriate sky frames and dividing by the flat fields. Target frames were then registered and combined to produce the final reduced images in each filter. $H$-band images of each field were constructed by adding the corresponding $\mathrm{CH}_{4 s}$ and $\mathrm{CH}_{4 l}$ images for the particular field in question.

Infrared sources were identified at $K_{s}$ band using the automated source extractor DAOFIND routine within IRAF (Stetson 1987). DAOFIND was run on each field using a FWHM of 2.8 pixels and a single pixel-finding threshold equal to three times the mean noise of each image. Each field was individually inspected, and the DAOFIND coordinate files were edited

\footnotetext{
7 http://www.cfa.harvard.edu/COMPLETE/data_html_pages/ OphA_Extn2MASS_F.html

8 IRAF is distributed by the National Optical Astronomy Observatory, which is operated by the Association of Universities for Research in Astronomy, Inc. under cooperative agreement with the National Science Foundation.
} 


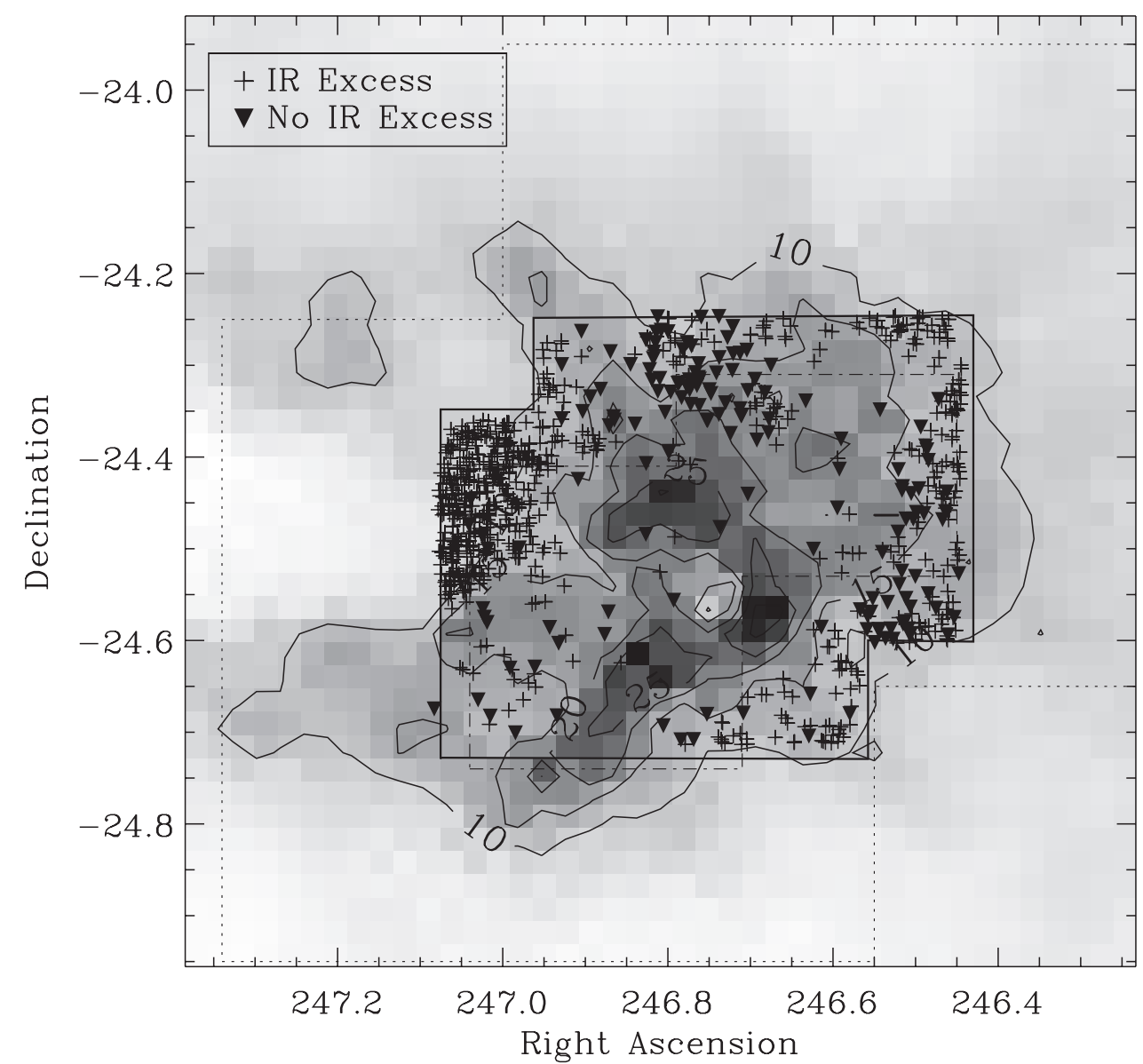

Figure 1. Plot of spatial distribution of our candidate $\rho$ Oph members (crosses: excess sources: filled triangles: non-excess sources) superposed on the $A_{V}$ contours from the COMPLETE project. $A_{V}$ contours are plotted and labeled at $A_{V}=10,15,20$, and 25 . Our $920 \operatorname{arcmin}^{2}$ survey area is indicated by the solid outline. The survey areas of Geers et al. and Alves de Oliveira et al. are indicated by the dot-dashed and dotted outlines, respectively.

to remove bad pixels and any objects misidentified as stars, as well as to add any missed stars to the list. Objects within $30^{\prime \prime}$ of the field edges were also removed from the list, as they were in low signal-to-noise regions of the image as a result of the dither pattern used. Aperture photometry was then performed on all fields in each filter using the PHOT routine within IRAF. An aperture of 4 pixels in radius was used for all target photometry, and a 10 pixel radius was used for the standard-star photometry. Sky values around each source were determined from the mode of intensities in an annulus with inner and outer radii of 10 and 20 pixels, respectively. Our choice of aperture size for our target photometry ensured that the individual source fluxes were not contaminated by the flux from neighboring stars; however, they are not large enough to include all the flux from a given source. In order to account for this missing flux, aperture corrections were determined using the MKAPFILE routine within IRAF. The instrumental magnitudes for all sources were corrected to account for the missing flux.

Photometric calibration was accomplished using the list of standard stars of Persson et al. (1998). The standards were observed on the same nights and through the same range of air masses as the $\rho$ Ophiuchi cloud. Zero points and extinction coefficients were established for each night. ${ }^{9}$ All magnitudes and colors were transformed to the California Institute of Technology (CIT) system using MKO to 2MASS and 2MASS

\footnotetext{
9 We found a zero-point offset of 1.8 mag through each filter.
}

to CIT photometric color transformation equations ${ }^{10}$ and the conversion relations of Stephens \& Leggett (2004). Because of the extensive spatial overlapping of the cloud images, a number of sources were observed at least twice. We compared the $J H K_{s}$ magnitudes of 200 duplicate stars identified in the overlap regions. For all stars brighter than the completeness limit of our survey, the photometry of the duplicate stars agreed to within 0.15 mag. Plots of our photometric errors as a function of magnitude are presented in Figure 2, for $J$ (left panel), $H$ (middle panel), and $K_{s}$ (right panel).

The completeness limit of our observations was determined by adding artificial stars at random positions to each of the 19 fields in all four filters and counting the number of sources recovered by DAOFIND. Artificial stars were added in 12 separate half-magnitude bins, covering a magnitude range of 16.00-22.00, with each bin containing 100 stars. The artificial stars were examined to ensure that they had the same FWHM as the real sources in each image. Aperture photometry was performed on all sources to confirm that the assigned magnitudes of the added sources agreed with those returned by PHOT. All photometry agreed to within 0.10 mag. DAOFIND and PHOT were then run and the number of identified artificial sources within each half-magnitude bin was tallied. This process was repeated 20 times. We estimate that our survey is $90 \%$ complete to $J=20.00, H=20.00$, and $K_{s}=18.50$. Furthermore, saturation of objects in each image occurred at $J \simeq 12.0$,

\footnotetext{
10 See http://www.astro.caltech.edu/ jmc/2mass/v3/transformations/.
} 

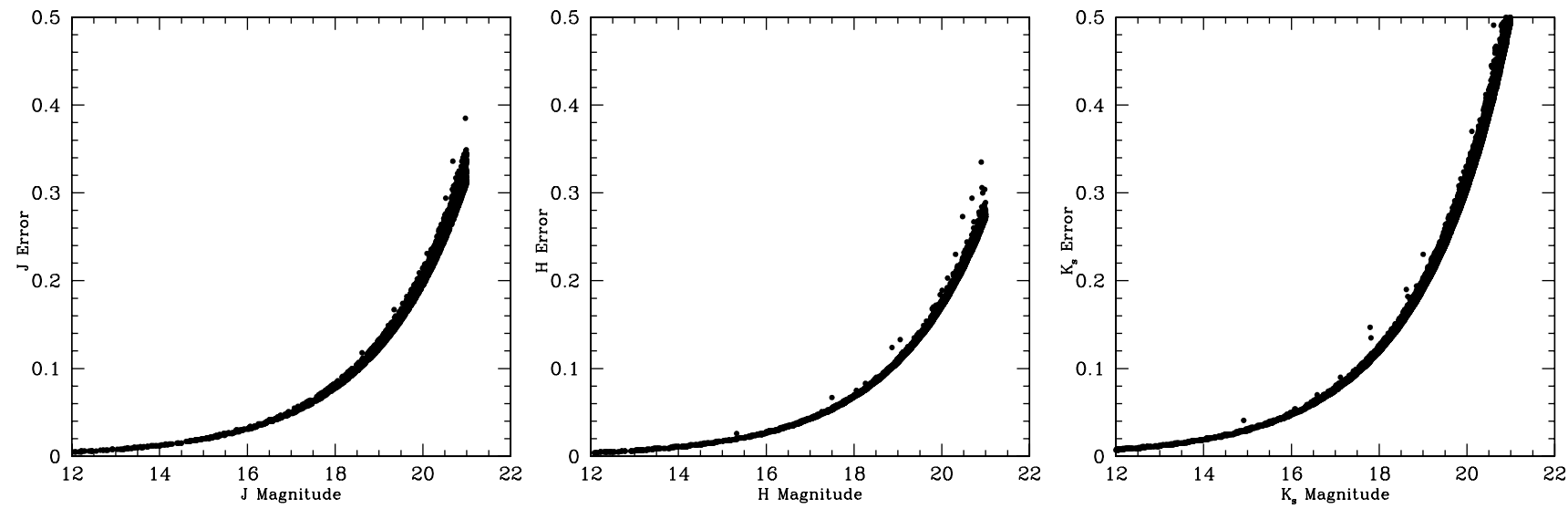

Figure 2. Photometric errors as a function of magnitude at $J$ (left panel), $H$ (middle panel), and $K_{s}$ (right panel). Completeness limits are at $J=20.0, H=20.0$, and $K_{s}=18.50$, as discussed in the text. Saturation limits are at $J \simeq 12.0, H \simeq 11.0$, and $K_{s} \simeq 10.0$.

$H \simeq 11.0$, and $K_{s} \simeq 10.0$, respectively. Thus, our observations are sensitive to $12.0 \leqslant J \leqslant 20.0,11.0 \leqslant H \leqslant 20.0$, and $10.0 \leqslant K_{s} \leqslant 18.5$, respectively.

Coordinates for all objects were determined relative to the positions of known objects in the $2 \mathrm{MASS}^{11}$ catalog. In particular, plate solutions were done using the 2MASS catalog in conjunction with WCSTools, a package of programs and a library of utility subroutines for setting and using the world coordinate system in the headers of the most common astronomical image formats to relate image pixels to sky coordinates. ${ }^{12}$ The resulting coordinates of all objects in our survey have typical rms uncertainties of $\sim 0^{\prime}$. 20 relative to the coordinates of previously known stars used in their determinations.

\section{RESULTS}

We detected a total of 2283 sources at all three wavelengths at or brighter than our $J H K_{s}$ completeness limits within the 920 $\operatorname{arcmin}^{2}$ region outlined by the solid black lines in Figure 1. Of course, many more sources were detected at each individual waveband. At $K_{s}$, we detected 7081 sources to $5 \sigma$ and 6882 to our $K_{s}=18.50$ completeness limit. We used the locations of the $K_{s}$ detections to search for counterparts at $H$ and $J$. Since we used the combination of the 30 minute on-source duration observations in the methane-on and methane-off filters to construct the $H$-band image, instead of just the 5 minutes total on-source integration times at $K_{s}$ and $J$ bands, we detected 7090 sources at $H$ to $5 \sigma$ and 6986 to our $H=20.0$ completeness limit. Finally, we detected just 3486 sources to $5 \sigma$ at $J$ and 2404 to our $J=20.00$ completeness limit, reflecting the fact that we were observing through the highest extinction portions of the $\rho$ Oph core.

Our survey boundaries are indicated in Figure 1, superposed on the extinction map (described in Section 2), displayed both in gray scale and by contour levels. From Figure 1, it is evident that our survey encompassed the highest extinction portions of the $\rho$ Oph cloud core. The vast majority of detected sources lie between the $A_{V}=5$ and $A_{V}=15$ contour levels, whereas much of the surveyed area has $A_{V} \geqslant 20$.

In Figure 3 (left panel), we present the $J-H$ versus $H-K_{s}$ color-color diagram for all 2283 objects with $10 \leqslant K_{s} \leqslant 18.5$

\footnotetext{
11 This publication makes use of data products from the Two Micron All-Sky Survey, which is a joint project of the University of Massachusetts and the Infrared Processing and Analysis Center/California Institute of Technology, funded by the National Aeronautics and Space Administration and the National Science Foundation.

12 http://tdc-www.harvard.edu/wcstools/
}

in our survey area, with available photometry at all three $\left(J H K_{s}\right)$ bands in our data. The greatest uncertainty in the colors is less than $0.2 \mathrm{mag}$ for all sources and is indicated by the size of the cross in each panel of Figure 3. The solid curve in each panel represents the locus of colors corresponding to unreddened main-sequence stars, ranging in spectral type from early $\mathrm{O}$ to M9, after converting the 2MASS colors to the CIT system. The locus of the colors of giant stars is represented by a dashed line in each panel (Bessell \& Brett 1988). The two parallel dashed lines define the reddening band for main-sequence stars and are parallel to the reddening vector. The classical T Tauri star (CTTS) locus in these diagrams extends from $J-H=0.81$, $H-K=0.50$ to $J-H=1.10, H-K_{s}=1.00$ (Meyer et al. 1997). A diagonal arrow representing the effect of 5 mag of visual extinction is also shown. The reddening law of Cohen et al. (1981), derived in the CIT system and having a slope of 1.692 , has been adopted.

Note the offset of the detected sources in the left panel of Figure 3 from the $(0,0)$ position in the color-color diagram, indicating that all sources suffer at least $A_{V}=5 \mathrm{mag}$ of visual extinction-confirming that we are looking through the darkest portion of the $\rho$ Oph cloud core. Of the 2283 stars plotted in the left panel of Figure 3, 1139 deredden to the CTTS locus. Therefore, these objects possess infrared excess emission and are referred to as "excess sources" in the following. Among the 1139 excess sources, 830 have available Spitzer photometry. We have divided the remaining sources into two groups. The first group consists of 709 "non-excess" sources-those that definitely would not deredden to the CTTS locus. The second group, consisting of 435 sources, would deredden to photospheric colors characteristic of very low mass stars or brown dwarfs of spectral types in the range $\mathrm{M} 7-\mathrm{L} 0$ as given by Luhman et al. (2010). Of the 709 sources that do not display infrared excesses, 533 have available Spitzer photometry. Of the 435 sources that would deredden to M7-L0 photospheric colors, 378 have available Spitzer photometry.

We have estimated the effective temperatures for the 1723 sources for which successful fits were obtained to both our $J H K_{s}$ photometry and the Spitzer mid-infrared photometry. Spitzer photometry is taken from either the c2d (the Spitzer "From Molecular Cores to Planet-Forming Disks" Legacy Program) CLOUDS catalog for L1688 ${ }^{13}$ or Gutermuth et al. (2009), for sources not present in the $\mathrm{c} 2 \mathrm{~d}$ catalog.

\footnotetext{
13 http://irsa.ipac.caltech.edu/data/SPITZER/C2D/clouds.html
} 

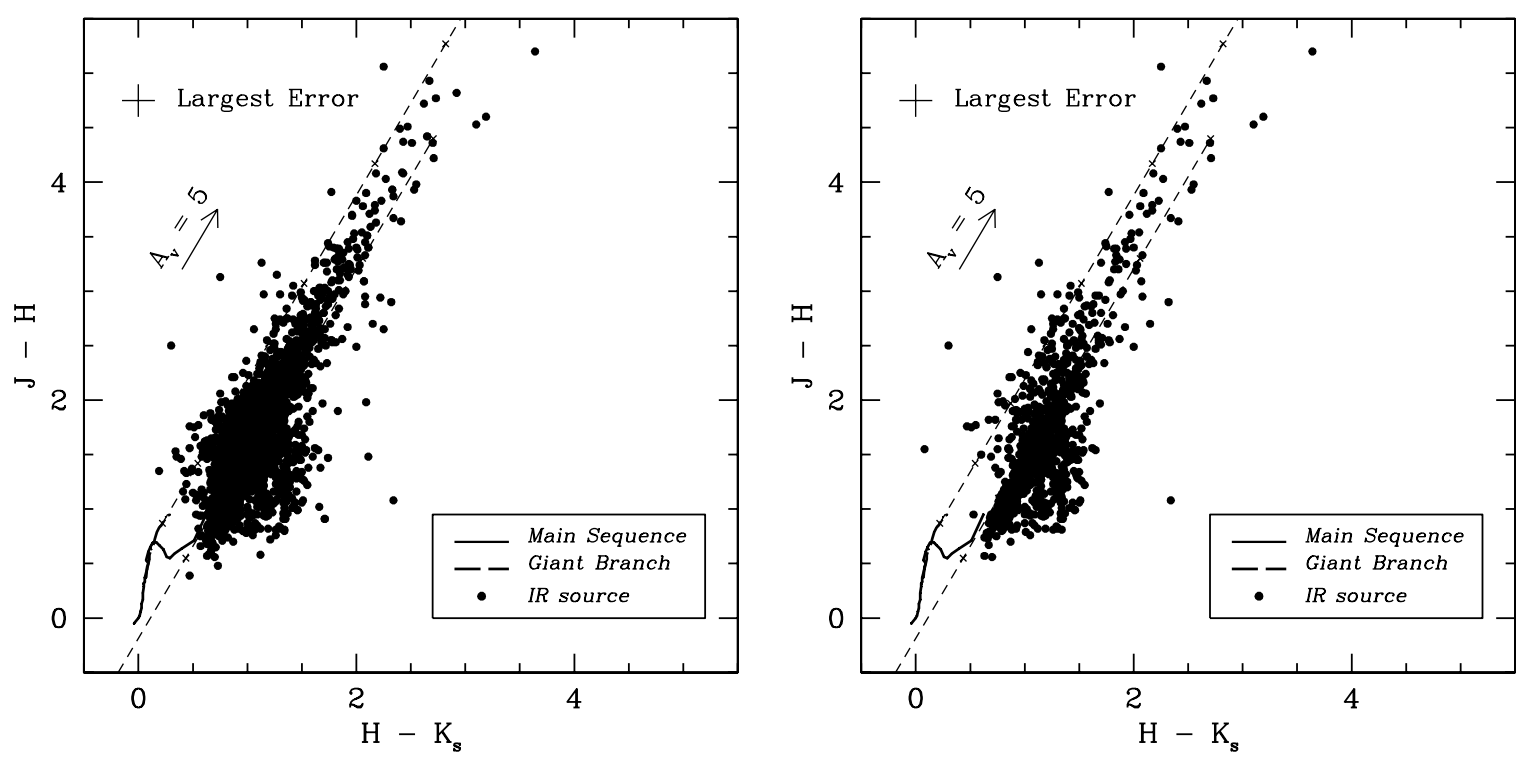

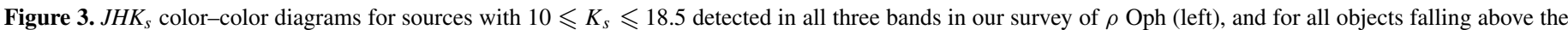

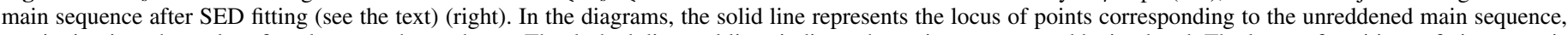

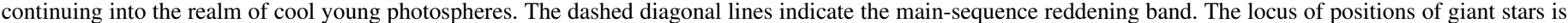

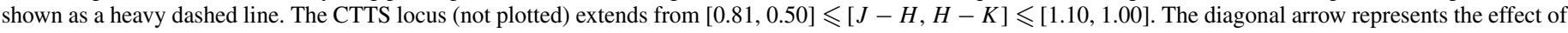
$5 \mathrm{mag}$ of visual extinction. The uncertainty in the colors for all sources is magnitude dependent, but always $\leqslant 0.2 \mathrm{mag}$, as labeled in both diagrams.

The combined NIR and Spitzer photometry for each source was fit with a model spectrum to estimate its effective temperature, $T_{\text {eff }}$. Four possible models were used for each source. These were (1) the 1 Myr COND models for $T_{\text {eff }} \leqslant 1500 \mathrm{~K}$ (Baraffe et al. 2003), (2) the 1 Myr DUSTY models for $1500 \mathrm{~K} \leqslant T_{\text {eff }} \leqslant 3000 \mathrm{~K}$ (Chabrier et al. 2000), (3) the NextGen models for $T_{\text {eff }} \geqslant 1700 \mathrm{~K}$, with solar gravity and metallicity (Hauschildt et al. 1999), or (4) blackbody spectra for all possible temperatures. Dereddened $K_{S}$ magnitudes (for $d=124 \mathrm{pc}$ ) were derived using the observed $K_{s}$ magnitudes and the $A_{V}$ estimates obtained by dereddening each source to either the main sequence (within the reddening band of Figure 3) or the CTTS locus (for sources to the right of the reddening band of Figure 3). Figure 3 (right panel) shows the distribution of sources in the $J H K_{s}$ color-color diagram that are found to lie above the main sequence from our SED fits.

In Figure 4, we present the dereddened $K_{s}$ magnitude as a function of estimated effective temperature for the 827 "excess" (top panel) and the 527 "non-excess" (middle panel) sources for which successful fits were obtained to their SEDs determined by our new $J H K_{s}$ data combined with Spitzer data. Objects in the cloud-exterior region, from the same off-cloud region as in Marsh et al. (2010b), are plotted in the bottom panel of Figure 4, for comparison. This bottom panel shows the results of fits to 509 off-cloud sources for which both deep $J H K_{s}$ and Spitzer photometry is available.

All three panels of Figure 4 show model curves for the $1 \mathrm{Myr}$ COND (dashed) and DUSTY (dotted) models and for mainsequence stars (solid) for an assumed distance of $124 \mathrm{pc}$. The $T_{\text {eff }}$ ranges used for the different atmospheric models are colorcoded and indicated at the bottom of each panel. Sources best fit using the 1 Myr COND models are plotted in red, those best fit using the 1 Myr DUSTY models are plotted in green, and those best fit using the NextGen models are plotted in mustard. In addition, fits to blackbodies of a specified temperature are plotted in blue.
Details of the fitting procedure are described in Marsh et al. (2010b), with the improvement in the present work that the Spitzer filter bandpasses have been convolved with the model atmospheres to derive Spitzer IRAC magnitudes for each model. For purposes of the model fitting described above, $A_{V}$ values were assigned to be those derived from dereddening each source to the CTTS locus of Figure 3, for the "excess" sources, and those derived by dereddening to the main sequence for the "nonexcess" sources, instead of letting $A_{V}$ be a free parameter.

In order to test the validity of our fitting procedure, we plot the locations assigned by our SED-fitting program to known, spectroscopically confirmed brown dwarfs in $\rho$ Oph from the tabulation of Geers et al. (2011). In Figure 4, the open diamonds represent known brown dwarfs with IR excesses (top panel) and known brown dwarfs without IR excesses (middle panel). In both cases, our fitting would independently determine these objects to lie above the main sequence and to have low values of $T_{\text {eff }}$.

\section{DISCUSSION}

\subsection{Candidate New Members and Their Properties}

The identification of the new candidate cloud members in $\rho$ Oph is primarily based on their location in the plots of Figure 4 . The top panel of Figure 4 shows a dramatically different distribution of sources from those in the middle and bottom panels. Note the dearth of reddened main-sequence stars in the top panel combined with the presence of disks, inferred from the preponderance of blackbody best fits. In the top panel of Figure 4, 764 of the 827 successfully fit infrared excess sources lie above the main sequence, identifying them as PMS objects and thus as candidate cluster members. The open diamonds plotted in the top panel of Figure 4 represent fits to the photometry of previously known and spectroscopically confirmed brown dwarf members of the cluster that also display infrared excess emission from disks, for comparison. 

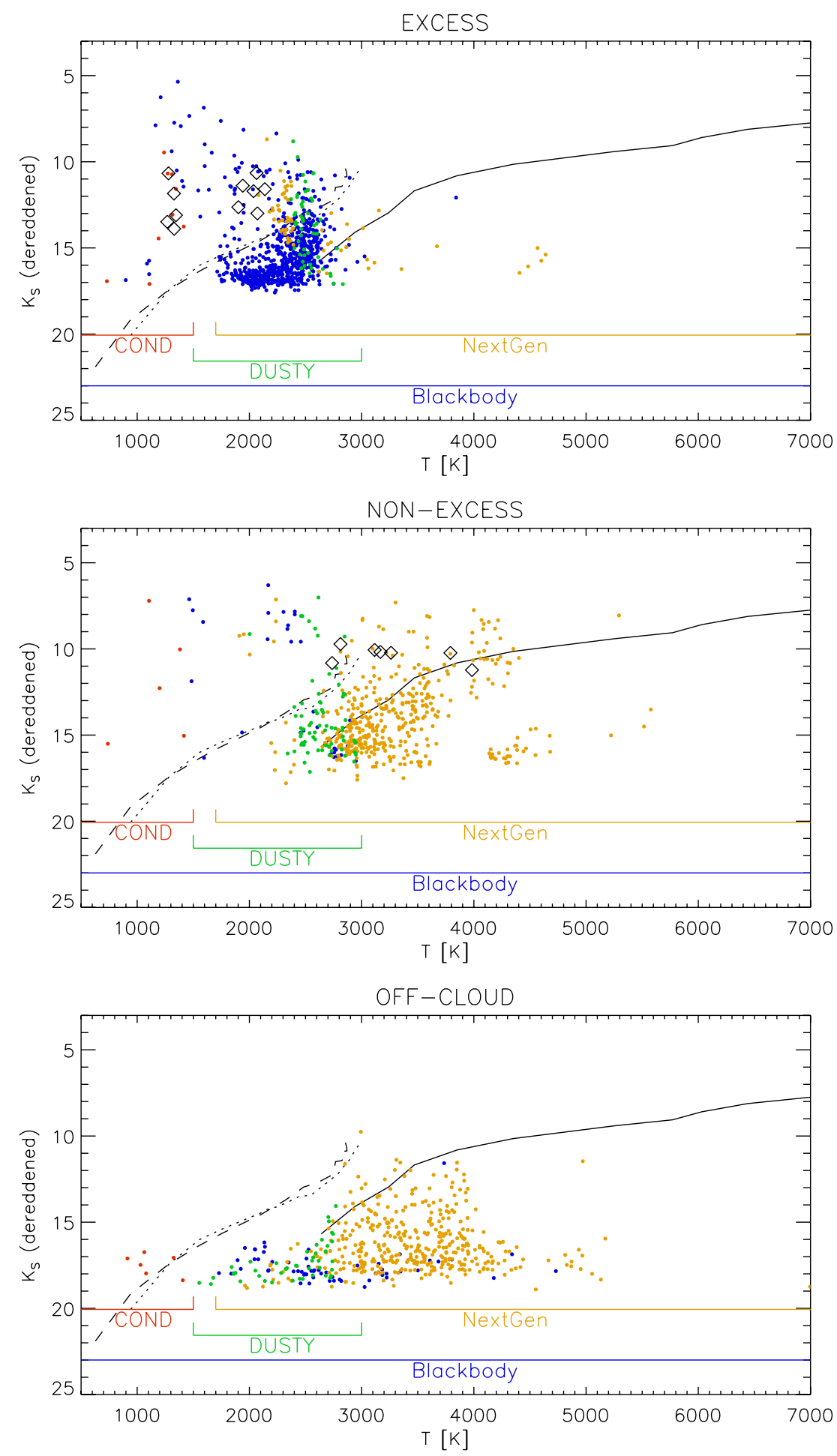

Figure 4. Dereddened $K_{s}$ magnitude as a function of estimated temperature for sources for which successful SED fits were obtained to their $J H K_{s}$ and $S p i t z e r$ IRAC data. In the top panel, we plot the locations of the 827 "excess" sources, along with the locations of known, spectroscopically confirmed brown dwarfs with IR excesses, which are indicated by open diamond symbols. In the middle panel, we plot the locations of 527 "non-excess" sources, along with the locations of spectroscopically confirmed brown dwarfs lacking IR excesses, which are indicated by the open diamond symbols. In the bottom panel, we plot the locations of sources located in the cloud-exterior region from Marsh et al. (2010b). Model curves are plotted in each panel for the 1 Myr COND (dashed) and DUSTY (dotted) models, as well as for the main-sequence NEXTGEN models (solid), for an assumed distance of $124 \mathrm{pc}$.

An artifact in the top panel of Figure 4 is the presence of a gap in the distribution of $T_{\text {eff }}$ values from the model fits in the $1200 \mathrm{~K} \leqslant T_{\text {eff }} \leqslant 1800 \mathrm{~K}$ range. The root cause of this gap is that objects surrounded by circumstellar material and therefore exhibiting SEDs characteristic of disks or late-stage protostars were fit to purely photospheric models (COND,
DUSTY, NextGen, blackbody). At the lowest temperatures $\left(T_{\text {eff }} \leqslant 1500 \mathrm{~K}\right)$, the COND models tend to give fairly flatlooking SEDs that often provide artificially good fits to flatspectrum protostars, while the more evolved YSOs are better fit by higher temperature models that are more Planck-like (DUSTY, NextGen, blackbody). It can be seen from the top 
Table 3

Rho Ophiuchi Low-mass Candidate Members

\begin{tabular}{|c|c|c|c|c|c|c|c|c|c|c|c|c|c|c|c|c|c|c|c|c|}
\hline ID & R.A. ${ }^{a}$ & Decl. $^{\mathrm{a}}$ & $J$ & $\sigma J$ & $H$ & $\sigma H$ & $K$ & $\sigma K$ & $I 1$ & $\sigma I 1$ & $I 2$ & $\sigma I 2$ & $I 3$ & $\sigma I 3$ & $I 4$ & $\sigma I 4$ & $A_{V}^{\mathrm{b}}$ & $T_{\text {eff }}{ }^{\mathrm{c}}$ & Model $^{\mathrm{d}}$ & $\overline{\text { IREX }^{\mathrm{e}}}$ \\
\hline 1 & $16: 25: 46.71$ & $-24: 18: 13.6$ & 17.92 & 0.07 & 16.48 & 0.04 & 15.31 & 0.04 & 0.351 & 0.034 & 0.254 & 0.023 & 0.418 & 0.327 & -1.150 & 1.540 & 3.3 & 2099 & B & $\mathrm{E}$ \\
\hline 2 & $16: 25: 46.74$ & $-24: 19: 15.9$ & 19.98 & 0.19 & 18.27 & 0.08 & 16.84 & 0.07 & 0.059 & 0.018 & 0.042 & 0.013 & 0.102 & 0.157 & 0.163 & 0.288 & 5.6 & 1959 & B & $\mathrm{E}$ \\
\hline 3 & $16: 25: 46.80$ & $-24: 19: 56.4$ & 19.29 & 0.13 & 17.80 & 0.06 & 16.83 & 0.07 & 0.110 & 0.022 & 0.061 & 0.027 & 0.328 & 0.247 & 0.850 & 0.462 & 5.6 & 2234 & B & $\mathrm{E}$ \\
\hline 4 & $16: 25: 46.84$ & $-24: 17: 58.5$ & 18.15 & 0.08 & 16.25 & 0.03 & 14.96 & 0.03 & 0.569 & 0.040 & 0.414 & 0.030 & 0.121 & 0.345 & 0.658 & 1.070 & 8.7 & 2401 & B & $\mathrm{E}$ \\
\hline 5 & $16: 25: 46.92$ & $-24: 20: 32.8$ & 19.46 & 0.16 & 18.01 & 0.07 & 17.00 & 0.07 & 0.078 & 0.024 & 0.049 & 0.014 & 0.078 & 0.164 & 0.172 & 0.324 & 4.8 & 2190 & B & $\mathrm{E}$ \\
\hline
\end{tabular}

Notes.

a Coordinates listed are J2000. Units of right ascension are hours, minutes, and seconds, and units of declination are degrees, arcminutes, and arcseconds.

${ }^{\mathrm{b}}$ Extinction estimates were calculated by dereddening each source in the $J H K$ color-color diagram as discussed in the text.

${ }^{c}$ Effective temperatures obtained from model fits as discussed in the text.

${ }^{\mathrm{d}}$ Model used for best fit. B: blackbody, D: DUSTY, C: COND, N: NextGen. See the text for details.

e Infrared excess (E) or non-excess (NE) source.

(This table is available in its entirety in a machine-readable form in the online journal. A portion is shown here for guidance regarding its form and content.)

panel of Figure 4 that the distinct gap in the distribution of sources in the $1200 \mathrm{~K} \leqslant T_{\text {eff }} \leqslant 1800 \mathrm{~K}$ range is not an artifact of the $1500 \mathrm{~K}$ boundary between the COND and DUSTY models - rather, this gap represents the temperature range over which none of the photospheric models can adequately mimic circumstellar disks.

A substantial fraction of the newly discovered population of "excess" sources plotted in the top panel of Figure 4 seems concentrated above the lowest $T_{\text {eff }}$ NextGen models, but below the COND/DUSTY models. This is very likely due to suppressed $K$-band flux due to extinction of the YSO photospheres by cool disk material (e.g., Mayne \& Harries 2010).

In the middle panel of Figure 4, we plot the 527 "non-excess" sources for which successful SED fits were obtained to our combined $J H K_{S}$ and available Spitzer photometry. Among these "non-excess" sources, most $(343 / 527)$ lie in the region below the main sequence at the cloud's distance in the dereddened $K_{s}$ versus $T_{\text {eff }}$ plot. Therefore, most of the non-excess sources are consistent with being background objects. However, 184 of the 527 non-excess sources plotted in the middle panel of Figure 4 lie above the main sequence and are candidate cloud members. The open diamonds plotted in the middle panel of Figure 4 show the locations of fits to the photometry of previously known, spectroscopically confirmed brown dwarf members of the cluster that lack infrared excess emission.

The bottom panel of Figure 4 shows the distribution of sources from an off-cloud region (same off-cloud region as used by Marsh et al. 2010b, to $5 \sigma$ detection limits of $J=20.5$, $H=20.0$, and $K_{s}=19.0$ ). The majority of objects detected in the off-cloud region lie below the locus of main-sequence photospheres at the distance to $\rho$ Oph. Sources falling below the main-sequence locus in Figure 4 are reddened background stars.

We therefore find a total of 948 candidate YSOs in the $\rho$ Ophiuchi cluster, of which 764 are excess sources and 184 are non-excess sources. Table 3 lists these sources. Column (1) of Table 3 is an ordinal source identification number, followed by each candidate object's $\alpha(2000)$ and $\delta(2000)$ coordinates. We then list our NIR photometry in the order, $J, \sigma_{J}, H, \sigma_{H}, K_{s}$, and $\sigma_{K_{s}}$, followed by the IRAC photometry with corresponding errors in each of the four IRAC bands $(3.6 \mu \mathrm{m}, 4.5 \mu \mathrm{m}$, $5.8 \mu \mathrm{m}$, and $8.0 \mu \mathrm{m}$, respectively) in ascending wavelength order. The next column lists the extinction values $\left(A_{V}\right)$ derived by dereddening each source to either the main sequence (for "non-excess" sources) or the CTTS locus (for "excess" sources) in the $J-H$ versus $H-K_{s}$ color-color diagram of Figure 3 . The next column lists the best-fit value of $T_{\text {eff }}$ derived from model fitting to the SED of each source. The penultimate column lists the best-fit model type used to derive the tabulated $T_{\text {eff }}$ value. The last column indicates whether an individual source is an "excess" source (E) or "non-excess" source (NE).

The right panel of Figure 3 shows a plot of the location of these 948 candidate cluster members in the $J-H$ versus $H-K_{s}$ color-color diagram. A large fraction, $764 / 948$ or $81 \%$, of our candidate members lie in the infrared excess region of the $J H K_{s}$ color-color diagram in Figure 3. Predictions from both observations and modeling suggest that this is what one would expect for excess emission from circumstellar disks (e.g., Lada \& Adams 1992; Meyer et al. 1997; Haisch et al. 2000). If the infrared excesses do indeed originate in circumstellar disks, then this strengthens their identification as a significant population of new low-mass YSOs in the $\rho$ Ophiuchi cloud.

Figure 5 shows fit results for the subset of the 435 sources that could be dereddened to very low mass stellar or brown dwarf colors in the $J-H$ versus $H-K_{s}$ color-color diagram of Figure 3, for which good SED fits could be obtained. The three panels of Figure 5 illustrate the fact that, for this sample, the fraction of objects inferred to be PMS and therefore to be potential cluster members varies greatly with their assumed, intrinsic, unreddened colors, or, equivalently, with their derived values of $A_{V}$. For these new fits, the a priori values of extinction were based on dereddening sources to the photospheric $J H K_{s}$ colors given for the indicated intrinsic spectral type by Luhman et al. (2010) in the $J-H$ versus $H-K_{s}$ diagram. Spitzer photometry was available for 378 of these 435 sources, and successful fits were obtained for 373 (top panel), 368 (middle panel), and 372 (bottom panel) sources. The top panel shows the results of assuming an intrinsic spectral type of M7 for all fitted sources. In this case, only 81/373 fitted sources would be inferred to lie above the main sequence. The middle panel shows the results of assuming an intrinsic spectral type of L0 for all fitted sources. In this case, 348/368 fitted sources would be inferred to lie above the main sequence. Finally, the bottom panel shows the results of assuming an intrinsic spectral type somewhere between M7 and L0, close to M9. In this case, $312 / 372$ well-fit sources would be inferred to lie above the main sequence. Due to this large variation in the inferred fraction of PMS sources depending on the assumed intrinsic spectral type of each source for sources lying in this narrow range of the $J-H$ versus $H-K_{s}$ color-color diagram, we do not yet include any of these among the new, low-mass, YSO population of $\rho$ 

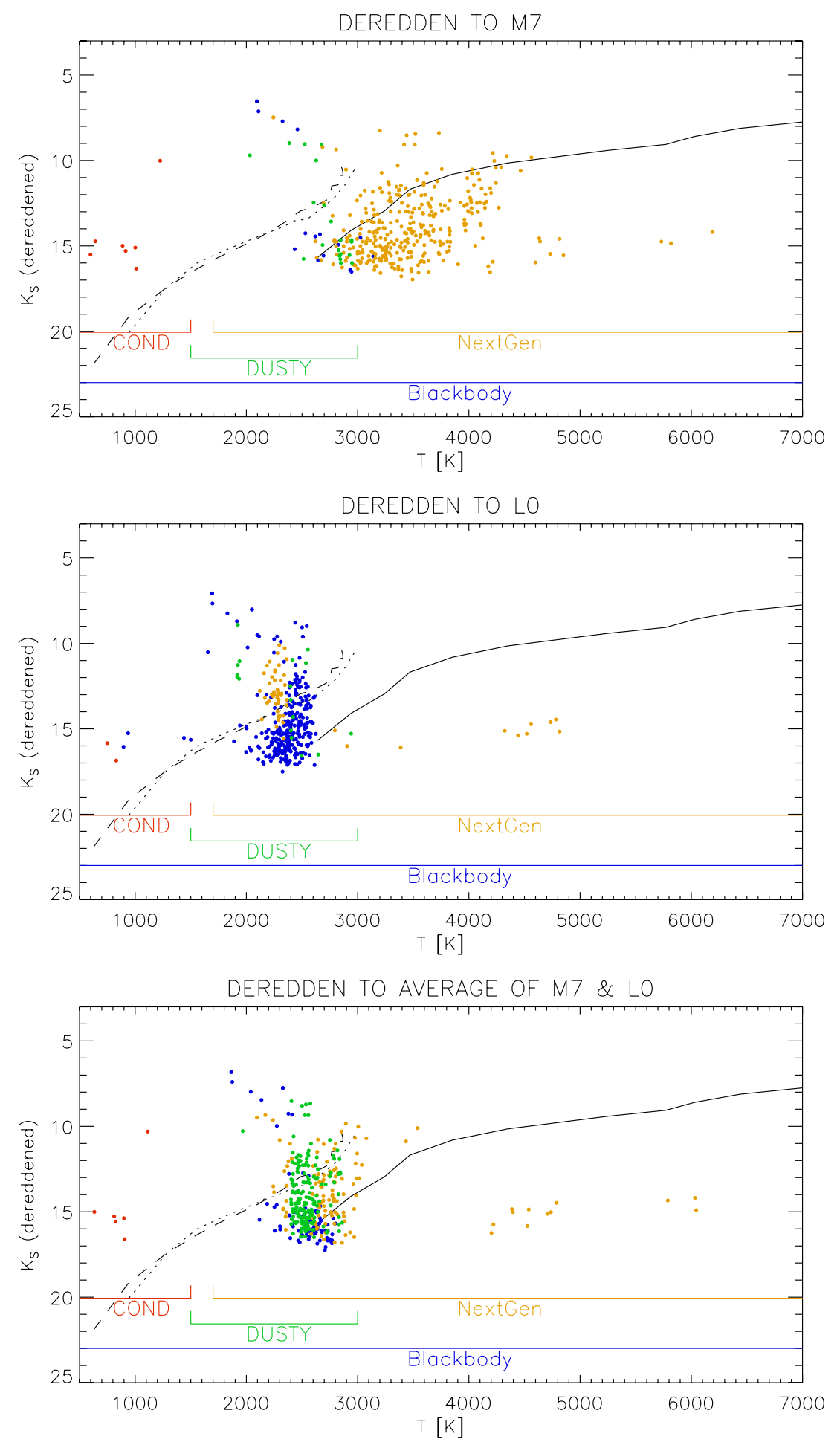

Figure 5. Same as Figure 4, except for "non-excess" sources that deredden to low-mass photospheric colors. The effect of assuming intrinsic $J-H$ vs. $H-K_{S}$ colors of M7 (top panel), L0 (middle panel), or to an average of M7-L0 spectral types (bottom panel) results in extinction values different enough to cause the demonstrated variation in the distribution of the best-fit SEDs in dereddened $K_{s}$ vs. $T_{\text {eff }}$ space.

Oph. Follow-up spectroscopy will reveal the intrinsic spectral types of this subset of objects and will determine what fraction are cloud members.

For our 948 candidate cloud members, however, we can estimate the range of masses to which this survey is potentially sensitive, given the distribution of $J H K_{s}$ brightnesses. At the low-mass end, our $J H K_{S}$ survey is sensitive to a bare photosphere with $T_{\text {eff }} \sim 1100 \mathrm{~K}$ for the $1 \mathrm{Myr}$ COND models at the distance to $\rho$ Oph, with no reddening, corresponding to $\sim 1.5 M_{\text {Jup }}$. This rises to 2.0, 4.0, and 8.5 $M_{\text {Jup }}$ for $A_{V}=5,10$, and 15, respectively. At the high-mass end, a source with $K_{s} \sim 14.0$ corresponds to an absolute $K_{s}$ of 8.52 or a $T_{\text {eff }} \sim 2250 \mathrm{~K}$-which is a late $\mathrm{M}$ spectral type. This $K_{s}$ magnitude corresponds to a mass of $10 M_{\text {Jup }}$ for the $1 \mathrm{Myr}$ COND model and rises to 15,35 , and $45 M_{\mathrm{Jup}}$ for $A_{V}=5,10$, and 15, respectively. However, these estimates do not take the complicating factor of disk excesses 


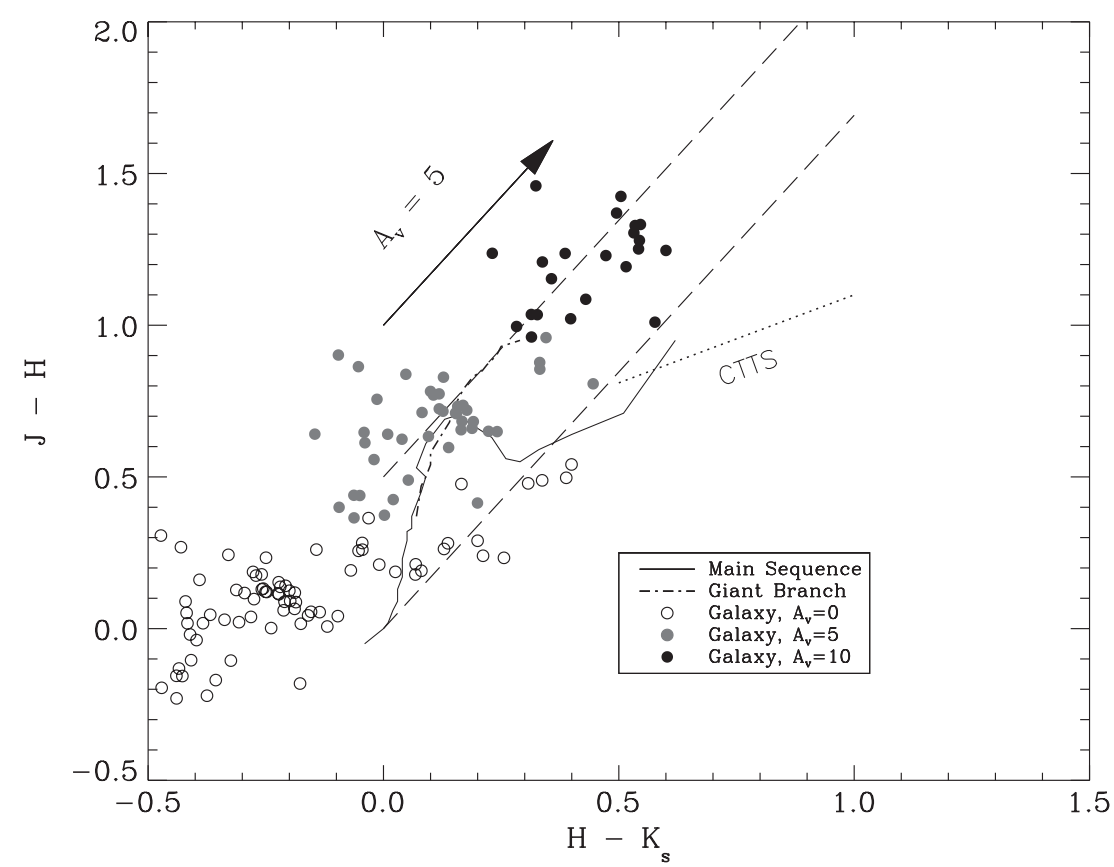

Figure 6. $J H K_{s}$ color-color diagrams for all galaxies from the GOODS-South field to our completeness limits of $J=20.0, H=20.0, K_{s}=18.50$ with no extinction (open circles), seen through $A_{V}=5$ (gray circles), and seen through $A_{V}=10$ (black circles). The loci of old main-sequence stars and substellar objects (solid curve), giants (dot-dashed line), and the classical T Tauri star (CTTS) locus (dotted line) are all shown for clarity. The parallel dashed lines delineate the reddening band-objects in this region deredden to main-sequence or giant colors. Comparison with Figure 3 shows that only a small fraction of background galaxies would fall into the "excess" region to the right of the rightmost reddening line.

into account. Spectroscopic follow-up is required to confirm the nature of individual objects and to better constrain their parameters. Because spectra for our candidate members are not available, it is not currently possible to derive a meaningful IMF for these objects. Nevertheless, the potential to determine the IMF for this cluster from $\sim 2 M_{\text {Jup }}$ through the substellar boundary is now a step closer.

\subsection{Contamination}

\subsubsection{Extragalactic}

We have completed a deep, wide-field, NIR imaging survey of the $\rho$ Ophiuchi cloud core to search for candidate low-mass member YSOs. Establishing membership of a given YSO in an SFR generally requires multi-wavelength observations, since multiple indicators of youth are required to establish membership for any individual candidate source. The candidate YSOs we have identified were selected based on fits to broadband SEDs. Many of our candidate objects display infrared excess emission, generally a good indicator of youth. Infrared excess by itself, however, may not always be definitive to establish membership of an individual source, since background active galactic nuclei (AGNs) could mimic YSO colors. However, given the high-extinction region to which our observations were limited, the effects of contamination by background galaxies, AGNs, or red giants are minimized, as demonstrated below.

An upper limit to the number of extragalactic contaminants among our candidate members can be obtained by contrasting the $K_{s}$ versus $T_{\text {eff }}$ plots for sources projected within the cloud core in Figure 1 (the "cloud" region) with those sources in the cloud "exterior" region. Here, the "exterior" region is the same one as defined in Marsh et al. (2010b). Using the list of spectroscopically confirmed brown dwarfs from Geers et al. (2011) as a guide for the location of cloud members in the dereddened $K_{s}$ versus $T_{\text {eff }}$ plot, and assuming that all of the inferred cluster members in the cloud "exterior" region in such a plot are spurious, an estimate of the (largest possible) number of extragalactic contaminants can be made in the following manner.

The number of objects that fall below the main sequence in the $K_{s}$ versus $T_{\text {eff }}$ plots of Figure 4 and are thus identified as background stars in the "cloud" (including the "excess" and "non-excess" sources) region is 404, whereas the corresponding number in the cloud "exterior" region is 385 . The numbers of sources above the main sequence in the "cloud" and "exterior" regions are 948 and 63, respectively. The number of contaminating sources in the "cloud" region can be predicted by scaling the number of "exterior" region objects that are above the main sequence (63) by the cloud:exterior background source count ratio, which is equal to 1.05 from the background source counts estimated above (404/385). Because background source counts are heavily affected by extinction, it is not appropriate to scale by the relative areas of the two regions. Rather, the scaling must be based on the number density ratio of extragalactic sources to background stars, which is the same for the "cloud" and "exterior" regions. Multiplying our cloud:exterior background source count ratio (1.05) by the exterior:cloud PMS source ratio (63/948), we find an upper limit to the percentage of contaminating sources among our candidate members of $\sim 7 \%$. Thus, 66 of our 948 candidate YSOs could be extragalactic background objects.

An alternative approach to estimating background contamination by extragalactic sources may be derived by inspection of Figure 6, in which we plot the $J H K$ colors of galaxies in the Great Observatories Origins Deep Survey (GOODS) South field imaged with VLT-ISAAC, after transforming the $J-H$ and $H-K_{s}$ colors to the CIT system. This makes Figure 6 directly comparable to Figure 3. The NIR GOODS-S data were acquired over a $\sim 160 \operatorname{arcmin}^{2}$ region (Retzlaff et al. 2010). The total 
number of galaxies is 76 in this field to our completeness limits. The GOODS-S field was observed through negligible extinction, whereas the minimum extinction toward our $920 \mathrm{arcmin}^{2}$ region is $A_{V}=5$ (see the text regarding Figure 3 in Section 3 ). Therefore, in Figure 6 we present plots of the appearance of the same 76 galaxies as if they were observed through $A_{V}=5$ (gray filled circles) and $A_{V}=10$ (black filled circles). Increasing the extinction has the dual effect of reddening and dimming these sources, such that only 42 galaxies and 22 galaxies, respectively, would be detected to our completion limits seen through $A_{V}=5$ and $A_{V}=10$. More importantly, the general population of background galaxies at these faint NIR magnitudes have very blue colors, so that they would not contaminate the "excess" region of the $\mathrm{JHK}_{\mathrm{S}}$ color-color plot.

\subsubsection{Galactic}

Red giants and faint red dwarfs are expected to be the major source of Galactic contaminants to our sample of candidate new, low-mass members of the $\rho$ Oph YSO population. Such contamination is minimized given the high extinction over most of our survey area, combined with its relatively high galactic latitude (16.377799 $\leqslant b \leqslant 17.153386)$.

An excellent estimate of Galactic contamination can be made directly from our modeling efforts. Of the 2283 total sources detected to our $J H K_{s}$ completeness limits, 1741 (including 830 "excess" and 530 "non-excess" sources) have available Spitzer photometry. Of these, 827 "excess" and 527 "nonexcess" sources could be successfully fit. Of the 827 successfully fit "excess" sources, plotted in the top panel of Figure 4, 61 fall below the main-sequence locus for an assumed distance of $124 \mathrm{pc}$, whereas of the 527 successfully fit "non-excess" sources, plotted in the middle panel of Figure 4, 343 fall below the main sequence. Thus, the total number of objects falling below the main sequence from our fits is 404 . This is our estimate of the number of Galactic background stars amongst the objects for which good fits to our $J H K_{s}$ and Spitzer photometry could be made. These Galactic contaminants are not counted amongst our 948 candidate YSOs.

\subsection{Comparison with Other Surveys}

A total of 316 verified (or candidate) members of the $\rho$ Ophiuchi cloud are listed in a recent review article (Wilking et al. 2008). Of these, 219 lie within our survey boundaries, and only 28 have $K_{s} \geqslant 14$. Of the 219 objects lying within our survey boundaries, 70 are saturated in our data at $K_{s}$ band, and a further 40 are undetected in our $J$-band data. This leaves 109 targets for which we have full $J H K_{s}$ and Spitzer IRAC photometry. All 109 were fit with our model-fitting algorithm, and 87 were found to lie above the main sequence. Of these 87 PMS sources, 57 lie in the "excess" region of the $J-H$ versus $H-K_{s}$ diagram, and 30 are in the "non-excess" region. This result demonstrates the efficacy of our modeling method at identifying PMS objects in an unbiased fashion, with regard to the presence or absence of disks.

Spitzer-selected objects, without available corresponding deep NIR photometry, are necessarily biased toward identifying sources with disks, often necessitating follow-up with X-ray telescopes to identify the "missing" diskless populations (e.g., Barrado et al. 2011; Pillitteri et al. 2010; Winston et al. 2010). Assuming the availability of sufficiently deep NIR and midIR photometry, our method presents an alternative approach to identifying the diskless population in nearby SFRs. This method also provides, for the first time, a uniform, unbiased means for identifying the entire PMS population in these regions, in a statistical sense.

Of course, selection biases are inherent in any observational effort - the aim is to understand what inherent biases there may be and to minimize their effect. Clearly, our method would not detect the two known Class 0 objects (VLA 1623 and IRAS 16253-2429) in L1688. Very faint, nearly edge-on disk systems might also be missed, due to lack of detection at $J$ band. Nevertheless, the efficacy of this method for detecting a heretofore undiscovered, significant new PMS population has been demonstrated, and the application of this method for uncovering new populations in other nearby SFRs is ongoing. Quantitative evaluation of selection biases inherent in this method vis-à-vis evolutionary stage, source orientation, and degree of embeddedness in the cloud awaits future work.

A comparison of our $J H K$ photometry with published photometry from the three recent, deep NIR surveys of $\rho$ Oph is presented in Table 4 . The coordinates of each source are listed first (as determined from our astrometry, described in Section 2 above), followed by our $J H K_{s}$ photometry. The next set of columns display Alves de Oliveira et al.'s (2010) source identifications and $J H K_{s}$ photometry for sources in common with our survey (from their Table 4). The next set of columns display Marsh et al.'s (2010b) source identifications and $J H K_{s}$ photometry for sources in common with our survey (from their Table 1). Finally, the last three columns of Table 4 display Geers et al.'s (2011) source identifications and $J K_{s}$ photometry for sources in common with our survey (from their Tables 1 and 2). Graphical presentations of these photometric comparisons are displayed in Figure 7.

Alves de Oliveira et al. (2010) list 110 candidate substellar objects, of which 74 fell within our survey area. We list photometry for all but two of these (their Source 16, which fell on bad pixels, and their Source 72, which lies on a bright diffraction spike in our images). The photometric agreement between the two data sets is generally good (see left column of Figure 7), with only Source 30 exhibiting highly discrepant values.

There are only eight sources in common between Alves de Oliveira's Table 4 and Marsh et al.'s Table 1. These correspond with Marsh et al.'s source nos. 829, 311, 654, 2978, 313, 222, 239 , and 334 . The rms error between the two sets of photometry for these sources, excluding Source 2978, is within 0.18 mag at $J, 0.12 \mathrm{mag}$ at $H$, and $0.090 \mathrm{mag}$ at $K_{s}$. For Marsh et al.'s Source 2978, the magnitude differences between the two sets of photometry are 2.66 at $J, 0.90$ at $H$, and 0.21 at $K_{s}$, with Alves de Oliveira's values always fainter. For the same source, our photometry varies from Marsh et al.'s by 0.29 at $H$ and 0.08 at $K_{s}$, with our values being the fainter ones (this source was not detected in our data at $J$ ). This photometric discrepancy may be due to the presence of extended faint nebulosity surrounding this object, as elaborated in the following for the case of the spectroscopically confirmed PMO, Marsh et al.'s Object 4450 (Marsh et al. 2010a).

For the seven candidate PMOs for which spectra were obtained by Marsh et al. (2010a), Alves de Oliveira state they found good agreement (between 0.02 and 0.23 mag difference) between the two sets of photometry at $K_{s}$ for Marsh et al.'s (2010b) source nos. 1449, 1307, 2438, and 2403, but differences of $0.4,1.42$, and $1.47 \mathrm{mag}$ at $K_{s}$ band for Marsh et al.'s source nos. 2974, 4450, and 3117, respectively. Unfortunately, Alves de Oliveira et al. did not publish the actual values for their $J H K_{s}$ magnitudes for any of these sources, except for the $J$ 
Table 4

Photometric Comparison with Recent Sensitive NIR Surveys of $\rho$ Oph

\begin{tabular}{|c|c|c|c|c|c|c|c|c|c|c|c|c|c|c|c|c|c|c|c|}
\hline \multicolumn{3}{|c|}{ R.A. (2000) } & \multicolumn{3}{|c|}{ Decl. (2000) } & \multicolumn{3}{|c|}{ This Work } & \multicolumn{4}{|c|}{ Alves de Oliveira et al. } & \multicolumn{4}{|c|}{ Marsh et al. } & \multicolumn{3}{|c|}{ Geers et al. } \\
\hline $\mathrm{h}$ & $\mathrm{m}$ & $\mathrm{s}$ & $\circ$ & ' & " & $J$ & $H$ & $K_{s}$ & $\begin{array}{c}\text { Source } \\
\text { No. }\end{array}$ & $J$ & $H$ & $K_{s}$ & $\begin{array}{c}\text { Source } \\
\text { No. }\end{array}$ & $J$ & $H$ & $K_{s}$ & $\begin{array}{c}\text { Source } \\
\text { No. }\end{array}$ & $J$ & $K_{s}$ \\
\hline 16 & 25 & 57.71 & -24 & 23 & 17.56 & 17.89 & 14.92 & 13.04 & 8 & 17.68 & 14.84 & 13.11 & & & & & & & \\
\hline 16 & 26 & 03.33 & -24 & 30 & 25.02 & 17.87 & 16.45 & 15.25 & 9 & 17.76 & 16.33 & 15.32 & & & & & & & \\
\hline 16 & 26 & 04.56 & -24 & 17 & 51.32 & 15.51 & 13.45 & 12.06 & & & & & & & & & 12 & 15.79 & 12.19 \\
\hline 16 & 26 & 07.24 & -24 & 21 & 16.49 & 20.90 & 18.47 & 16.61 & 10 & 21.74 & 18.60 & 16.69 & & & & & & & \\
\hline 16 & 26 & 07.92 & -24 & 17 & 22.89 & $\geqslant 21.00$ & 18.90 & 17.16 & 11 & 22.26 & 19.25 & 17.31 & & & & & & & \\
\hline 16 & 26 & 11.69 & -24 & 24 & 30.98 & $\geqslant 21.00$ & 18.17 & 16.13 & 13 & 21.20 & 18.45 & 16.13 & & & & & & & \\
\hline 16 & 26 & 13.16 & -24 & 19 & 09.71 & $\geqslant 21.00$ & 18.26 & 16.38 & 14 & 22.17 & 18.77 & 16.40 & & & & & & & \\
\hline 16 & 26 & 16.27 & -24 & 39 & 30.50 & 15.95 & 14.31 & 13.17 & 15 & 15.79 & 14.15 & 13.11 & & & & & & & \\
\hline 16 & 26 & 18.62 & -24 & 29 & 52.96 & 17.24 & 15.13 & 13.55 & & & & & & & & & 1 & 17.36 & 13.61 \\
\hline 16 & 26 & 18.89 & -24 & 26 & 10.95 & 14.85 & 13.25 & 12.06 & & & & & & & & & 13 & 14.84 & 12.14 \\
\hline 16 & 26 & 19.06 & -24 & 41 & 31.15 & 17.69 & 15.97 & 14.75 & 17 & 17.44 & 15.80 & 14.61 & & & & & & & \\
\hline 16 & 26 & 19.26 & -24 & 27 & 43.99 & 18.90 & 17.33 & 16.03 & 18 & 18.92 & 17.16 & 16.04 & & & & & & & \\
\hline 16 & 26 & 21.46 & -24 & 26 & 00.76 & 12.43 & 11.53 & 10.73 & & & & & & & & & 14 & 12.57 & 10.92 \\
\hline 16 & 26 & 22.25 & -24 & 37 & 08.27 & 18.98 & 16.88 & 15.64 & 19 & 19.15 & 17.10 & 15.86 & & & & & & & \\
\hline 16 & 26 & 22.20 & -24 & 24 & 06.58 & 16.32 & 14.83 & 13.64 & & & & & & & & & 2 & 16.70 & 13.94 \\
\hline 16 & 26 & 23.84 & -24 & 18 & 28.30 & 16.06 & 14.64 & 13.40 & & & & & & & & & 16 & 16.07 & 13.58 \\
\hline 16 & 26 & 24.24 & -24 & 15 & 52.53 & $17.53^{\mathrm{a}}$ & $15.48^{\mathrm{a}}$ & $14.02^{\mathrm{a}}$ & & & & & & & & & & & \\
\hline 16 & 26 & 24.32 & -24 & 15 & 48.07 & 18.60 & 15.90 & 13.75 & 21 & $18.78^{a}$ & $15.85^{\mathrm{a}}$ & $13.83^{\mathrm{a}}$ & & & & & & & \\
\hline 16 & 26 & 25.04 & -24 & 41 & 33.51 & 17.23 & 15.69 & 14.61 & 22 & 17.03 & 15.52 & 14.06 & & & & & & & \\
\hline 16 & 26 & 25.64 & -24 & 37 & 27.81 & 18.33 & 16.59 & 15.39 & & & & & 1045 & 18.201 & 16.544 & 15.319 & & & \\
\hline 16 & 26 & 25.98 & -24 & 33 & 13.87 & $\geqslant 21.00$ & 20.75 & 18.57 & 23 & 22.20 & 19.39 & 17.11 & & & & & & & \\
\hline 16 & 26 & 26.44 & -24 & 33 & 04.86 & $\geqslant 21.00$ & 18.69 & 16.92 & 24 & 22.13 & 19.45 & 17.45 & & & & & & & \\
\hline 16 & 26 & 27.76 & -24 & 26 & 41.60 & 14.04 & 12.91 & 11.96 & & & & & & & & & 17 & 14.26 & 12.09 \\
\hline 16 & 26 & 33.83 & -24 & 18 & 52.96 & $\geqslant 21.00$ & 20.91 & 18.84 & 25 & 22.42 & 19.31 & 17.39 & & & & & & & \\
\hline 16 & 26 & 34.00 & -24 & 35 & 55.88 & $\geqslant 21.00$ & 19.44 & 17.47 & 26 & 22.45 & 19.31 & 17.38 & & & & & & & \\
\hline 16 & 26 & 35.37 & -24 & 30 & 11.15 & $\geqslant 21.00$ & 18.01 & 15.93 & 27 & 22.32 & 18.35 & 15.94 & & & & & & & \\
\hline 16 & 26 & 35.31 & -24 & 42 & 40.93 & 19.31 & 17.72 & 16.28 & 28 & 19.68 & 17.79 & 16.36 & & & & & & & \\
\hline 16 & 26 & 36.00 & -24 & 20 & 58.65 & 18.72 & 15.77 & 13.69 & 29 & 18.84 & 15.65 & 13.46 & & & & & & & \\
\hline 16 & 26 & 36.82 & -24 & 18 & 59.99 & 17.00 & 16.06 & 15.23 & 30 & 16.24 & 14.35 & 13.06 & & & & & 3 & 17.01 & 14.55 \\
\hline 16 & 26 & 37.84 & -24 & 39 & 03.20 & 14.85 & 13.73 & 12.74 & 31 & 14.65 & 13.45 & 12.66 & & & & & 18 & 14.98 & 12.85 \\
\hline 16 & 26 & 38.78 & -24 & 23 & 22.20 & 14.94 & 12.81 & 11.42 & & & & & & & & & $2-3^{\mathrm{e}}$ & 15.10 & saturated \\
\hline 16 & 26 & 39.61 & -24 & 18 & 02.90 & $\geqslant 21.00$ & 18.67 & 17.18 & 32 & 22.55 & 19.15 & 17.11 & & & & & & & \\
\hline 16 & 26 & 39.68 & -24 & 22 & 07.51 & 18.44 & 17.04 & 15.93 & 33 & 18.16 & 16.74 & 15.68 & & & & & & & \\
\hline 16 & 26 & 39.92 & -24 & 22 & 32.43 & 16.19 & 14.66 & 13.43 & 34 & 15.95 & 14.53 & & & & & & & & \\
\hline 16 & 26 & 40.03 & -24 & 28 & 07.37 & $\geqslant 21.00$ & 18.32 & 16.58 & 35 & 21.45 & 18.56 & 16.52 & & & & & & & \\
\hline 16 & 26 & 40.58 & -24 & 24 & 27.26 & 20.71 & 18.25 & 15.71 & 36 & 21.60 & 18.37 & 16.69 & & & & & & & \\
\hline 16 & 26 & 40.85 & -24 & 30 & 50.62 & 17.44 & 14.89 & 13.12 & 37 & 17.32 & 14.77 & 13.18 & & & & & & & \\
\hline 16 & 26 & 41.80 & -24 & 36 & 11.50 & $\geqslant 21.00$ & 20.16 & 17.77 & 38 & 22.12 & 18.62 & 16.28 & & & & & & & \\
\hline 16 & 26 & 41.83 & -24 & 23 & 43.62 & $\geqslant 21.00$ & 18.62 & 17.04 & 39 & 22.59 & 19.32 & 17.07 & & & & & & & \\
\hline 16 & 26 & 42.73 & -24 & 24 & 27.15 & 19.66 & 15.57 & 13.15 & 40 & 19.44 & 15.59 & 13.22 & & & & & & & \\
\hline 16 & 26 & 43.78 & -24 & 24 & 50.95 & $\geqslant 21.00$ & 17.41 & 14.73 & 41 & 21.67 & 17.50 & 14.76 & & & & & & & \\
\hline 16 & 26 & 48.45 & -24 & 28 & 36.12 & 19.16 & 15.23 & 12.70 & 43 & 19.31 & 15.19 & 12.66 & & & & & & & \\
\hline 16 & 26 & 48.75 & -24 & 26 & 25.80 & 19.69 & 15.32 & 12.89 & 44 & 19.32 & 15.32 & 12.92 & & & & & & & \\
\hline 16 & 26 & 50.91 & -24 & 26 & 07.67 & $\geqslant 21.00$ & 19.14 & 17.12 & 45 & 21.73 & 19.17 & 17.27 & & & & & & & \\
\hline 16 & 26 & 51.22 & -24 & 32 & 41.43 & 15.02 & 14.46 & 13.76 & & & & & & & & & 19 & 15.30 & 13.89 \\
\hline 16 & 26 & 51.91 & -24 & 30 & 38.62 & $\geqslant 21.00$ & 16.32 & 13.33 & 46 & 21.30 & 16.43 & 13.45 & & & & & & & \\
\hline 16 & 26 & 52.70 & -24 & 24 & 52.85 & $\geqslant 21.00$ & 18.30 & 15.78 & 48 & 21.76 & 18.24 & 15.80 & & & & & & & \\
\hline 16 & 26 & 53.43 & -24 & 32 & 35.67 & 20.88 & 16.65 & 13.29 & 49 & 21.83 & 16.69 & 13.30 & & & & & & & \\
\hline 16 & 26 & 54.33 & -24 & 24 & 38.59 & $\geqslant 21.00$ & 17.16 & 14.02 & 50 & 21.71 & 17.25 & 14.04 & & & & & & & \\
\hline 16 & 26 & 54.74 & -24 & 27 & 02.40 & 17.85 & 14.76 & 12.69 & 51 & 17.92 & 14.91 & 12.87 & & & & & & & \\
\hline 16 & 26 & 55.34 & -24 & 21 & 17.10 & $\geqslant 21.00$ & 18.37 & 17.23 & & & & & 4220 & 19.771 & 18.361 & 17.317 & & & \\
\hline 16 & 26 & 55.47 & -24 & 28 & 22.42 & 19.85 & 17.21 & 15.54 & & & & & 1518 & 19.105 & 17.075 & 15.601 & & & \\
\hline 16 & 26 & 56.24 & -24 & 16 & 18.05 & 14.34 & 12.53 & 11.69 & & & & & 108 & 14.373 & 12.424 & 11.615 & & & \\
\hline 16 & 26 & 56.25 & -24 & 21 & 30.90 & $\geqslant 21.00$ & 18.75 & 17.34 & & & & & 4795 & 20.573 & 19.041 & 17.381 & & & \\
\hline 16 & 26 & 56.32 & -24 & 42 & 38.10 & 17.53 & 16.55 & 15.70 & & & & & 1254 & 17.753 & 16.633 & 15.865 & $2-1^{\mathrm{e}}$ & 17.68 & 15.53 \\
\hline 16 & 26 & 56.36 & -24 & 41 & 19.85 & 18.63 & 16.42 & 14.91 & 53 & 18.60 & 16.34 & 14.92 & 829 & 18.567 & 16.309 & 14.981 & 4 & 18.70 & 14.77 \\
\hline 16 & 26 & 56.87 & -24 & 28 & 36.33 & 18.20 & 14.72 & 12.75 & & & & & 233 & 18.256 & 14.690 & 12.800 & & & \\
\hline 16 & 26 & 57.34 & -24 & 35 & 38.11 & 19.30 & 15.22 & 12.79 & & & & & 236 & 19.198 & 15.111 & 12.782 & & & \\
\hline 16 & $\begin{array}{l}20 \\
26\end{array}$ & 57.37 & $\begin{array}{l}-24 \\
-24\end{array}$ & 42 & $\begin{array}{l}50.11 \\
18.70\end{array}$ & 18.86 & 17.15 & 15.99 & & & & & & & & & $2-2^{\mathrm{e}}$ & 18.94 & 15.73 \\
\hline 16 & 26 & 58.43 & -24 & 20 & 03.73 & $\geqslant 21.00$ & 18.13 & 16.86 & 54 & 20.55 & 18.12 & 16.68 & & & & & & & \\
\hline 16 & 26 & 58.35 & -24 & 21 & 30.28 & 16.09 & 13.10 & 11.61 & & & & & 115 & 16.299 & 13.178 & 11.515 & & & \\
\hline 16 & 26 & 58.67 & -24 & 24 & 55.47 & 20.25 & 17.25 & 14.91 & 55 & 20.22 & 17.15 & 14.84 & & & & & 5 & 19.64 & 15.30 \\
\hline 16 & 26 & 59.06 & -24 & 35 & 56.54 & 17.15 & 14.00 & 12.08 & & & & & 141 & 16.459 & 13.464 & 11.882 & 6 & 16.51 & 12.21 \\
\hline 16 & 26 & 59.94 & -24 & 24 & 21.62 & $\geqslant 21.00$ & 19.38 & 18.39 & & & & & 7614 & 20.740 & 19.286 & & & & \\
\hline
\end{tabular}


Table 4

(Continued)

\begin{tabular}{|c|c|c|c|c|c|c|c|c|c|c|c|c|c|c|c|c|c|c|c|}
\hline \multicolumn{3}{|c|}{ R.A. (2000) } & \multicolumn{3}{|c|}{ Decl. (2000) } & \multicolumn{3}{|c|}{ This Work } & \multicolumn{4}{|c|}{ Alves de Oliveira et al. } & \multicolumn{4}{|c|}{ Marsh et al. } & \multicolumn{3}{|c|}{ Geers et al. } \\
\hline $\mathrm{h}$ & $\mathrm{m}$ & $\mathrm{s}$ & $\circ$ & ' & " & $J$ & $H$ & $K_{s}$ & $\begin{array}{c}\text { Source } \\
\text { No. }\end{array}$ & $J$ & $H$ & $K_{s}$ & $\begin{array}{c}\text { Source } \\
\text { No. }\end{array}$ & $J$ & $H$ & $K_{s}$ & $\begin{array}{c}\text { Source } \\
\text { No. }\end{array}$ & $J$ & $K_{s}$ \\
\hline 16 & 27 & 01.91 & -24 & 22 & 06.47 & 20.03 & 15.62 & 13.48 & & & & & 291 & 19.853 & 15.573 & 13.196 & & & \\
\hline 16 & 27 & 02.99 & -24 & 26 & 14.68 & $\geqslant 21.00$ & 15.72 & 12.70 & & & & & 207 & $\ldots$ & 15.673 & 12.559 & & & \\
\hline 16 & 27 & 03.57 & -24 & 20 & 05.11 & 17.32 & 15.13 & 13.97 & 56 & 17.13 & 15.01 & 13.72 & 311 & 17.067 & 14.738 & 14.437 & & & \\
\hline 16 & 27 & 04.09 & -24 & 28 & 30.23 & 16.43 & 13.03 & 10.92 & & & & & 89 & 16.553 & 13.023 & 10.889 & & & \\
\hline 16 & 27 & 04.54 & -24 & 19 & 44.24 & $\geqslant 21.00$ & 18.56 & 17.71 & & & & & 5598 & 20.239 & 18.642 & 17.607 & & & \\
\hline 16 & 27 & 04.56 & -24 & 27 & 15.21 & 16.98 & 13.47 & 11.47 & & & & & 103 & 16.464 & 13.076 & 11.185 & & & \\
\hline 16 & 27 & 05.64 & -24 & 40 & 12.85 & 20.94 & 16.72 & 14.01 & & & & & 439 & 21.353 & 16.645 & 13.911 & & & \\
\hline 16 & 27 & 05.93 & -24 & 18 & 40.18 & 17.34 & 15.94 & 15.05 & 58 & 17.05 & 15.77 & 14.85 & 654 & 17.307 & 15.784 & 14.816 & & & \\
\hline 16 & 27 & 05.97 & -24 & 28 & 36.73 & 16.86 & 14.58 & 13.09 & & & & & & & & & 20 & 16.64 & 12.97 \\
\hline 16 & 27 & 05.98 & -24 & 16 & 14.15 & 18.76 & 17.21 & 16.19 & & & & & 1344 & 18.889 & 17.069 & 16.032 & & & \\
\hline 16 & 27 & 06.52 & -24 & 18 & 32.18 & 20.19 & 18.35 & 17.63 & & & & & 6412 & 19.965 & 18.600 & & & & \\
\hline 16 & 27 & 06.62 & -24 & 41 & 49.86 & 12.27 & 11.43 & 10.68 & & & & & 60 & 12.400 & 11.345 & 10.709 & & & \\
\hline 16 & 27 & 07.00 & -24 & 31 & 05.84 & $\geqslant 21.00$ & 18.50 & 15.94 & & & & & 1558 & 21.587 & 18.758 & 15.909 & & & \\
\hline 16 & 27 & 07.68 & -24 & 34 & 03.04 & $\geqslant 21.00$ & 18.63 & 16.75 & 59 & 22.33 & 19.00 & 16.58 & & & & & & & \\
\hline 16 & 27 & 08.03 & -24 & 20 & 06.87 & 17.83 & 14.99 & 13.63 & & & & & 312 & 17.768 & 14.851 & 13.397 & & & \\
\hline 16 & 27 & 08.05 & -24 & 31 & 42.32 & $\geqslant 21.00$ & 19.18 & 18.12 & & & & & 7145 & 19.906 & 18.942 & 18.168 & & & \\
\hline 16 & 27 & 08.14 & -24 & 41 & 18.94 & 20.49 & 18.40 & 17.13 & & & & & 3253 & 19.696 & 18.467 & 16.800 & & & \\
\hline 16 & 27 & 08.22 & -24 & 42 & 29.97 & 15.25 & 12.31 & 10.81 & & & & & 74 & 15.393 & 12.261 & $9.656^{\mathrm{b}}$ & & & \\
\hline 16 & 27 & 08.44 & -24 & 16 & 19.61 & 20.44 & 18.30 & 17.17 & & & & & 4933 & 20.158 & 18.726 & 17.468 & & & \\
\hline 16 & 27 & 09.01 & -24 & 30 & 25.31 & $\geqslant 21.00$ & 18.61 & 16.02 & 60 & 22.14 & 19.30 & 16.15 & & & & & & & \\
\hline 16 & 27 & 09.33 & -24 & 24 & 04.58 & 20.97 & 18.47 & 17.19 & & & & & 4788 & 19.401 & 18.166 & 17.591 & & & \\
\hline 16 & 27 & 09.36 & -24 & 32 & 15.24 & $\geqslant 21.00$ & 18.92 & 16.95 & & & & & 2438 & 19.701 & 18.018 & 16.741 & & & \\
\hline 16 & 27 & 09.59 & -24 & 24 & 17.93 & $\geqslant 21.00$ & 18.59 & 18.17 & & & & & 5710 & 19.606 & 18.248 & 18.566 & & & \\
\hline 16 & 27 & 09.80 & -24 & 34 & 41.27 & $\geqslant 21.00$ & 20.45 & 18.15 & 61 & 22.44 & 18.84 & 16.51 & & & & & & & \\
\hline 16 & 27 & 10.05 & -24 & 29 & 13.55 & 16.47 & 15.16 & 14.05 & 62 & 16.55 & 15.14 & 14.23 & & & & & & & \\
\hline 16 & 27 & 10.20 & -24 & 35 & 45.89 & $\geqslant 21.00$ & 18.63 & 17.13 & 63 & 22.01 & 18.92 & 17.10 & & & & & & & \\
\hline 16 & 27 & 10.33 & -24 & 33 & 22.32 & 17.32 & 13.92 & 11.92 & & & & & 147 & 17.401 & 13.854 & 11.910 & & & \\
\hline 16 & 27 & 11.28 & -24 & 23 & 27.15 & $\geqslant 21.00$ & 18.81 & 17.78 & & & & & 4863 & 19.503 & 18.568 & 17.460 & & & \\
\hline 16 & 27 & 11.60 & -24 & 23 & 21.80 & 20.31 & 18.16 & 17.19 & & & & & 4195 & 19.855 & 18.551 & 17.405 & & & \\
\hline 16 & 27 & 11.64 & -24 & 23 & 42.28 & 14.40 & 11.84 & 10.48 & & & & & 58 & 14.283 & 11.616 & 10.089 & & & \\
\hline 16 & 27 & 11.95 & -24 & 26 & 46.61 & $\geqslant 21.00$ & 18.81 & 17.99 & & & & & 5820 & 20.136 & 18.657 & 17.925 & & & \\
\hline 16 & 27 & 12.71 & -24 & 32 & 0.00 & $\geqslant 21.00$ & 19.26 & 18.20 & & & & & 14250 & 19.573 & 18.885 & 18.300 & & & \\
\hline 16 & 27 & 13.01 & -24 & 31 & 59.99 & $\geqslant 21.00$ & 18.67 & 16.92 & 64 & 22.13 & 19.28 & 17.05 & 2978 & 19.474 & 18.380 & 16.840 & & & \\
\hline 16 & 27 & 13.17 & -24 & 23 & 47.60 & $\geqslant 21.00$ & 17.67 & 15.53 & 65 & 20.88 & 17.60 & 15.67 & & & & & & & \\
\hline 16 & 27 & 13.55 & -24 & 34 & 14.43 & $\geqslant 21.00$ & 19.57 & 18.21 & & & & & 9002 & 20.211 & 19.772 & 18.713 & & & \\
\hline 16 & 27 & 13.99 & -24 & 32 & 06.19 & $\geqslant 21.00$ & 18.94 & 17.62 & & & & & 5076 & 20.054 & 19.112 & 17.741 & & & \\
\hline 16 & 27 & 14.08 & -24 & 22 & 50.59 & 20.98 & 18.26 & 16.58 & & & & & 2956 & 20.144 & 18.405 & 16.759 & & & \\
\hline 16 & 27 & 14.31 & -24 & 31 & 31.85 & 18.36 & 16.38 & 15.02 & 66 & 18.42 & 16.53 & 15.30 & & & & & & & \\
\hline 16 & 27 & 15.73 & -24 & 38 & 43.68 & 14.17 & 11.62 & 10.17 & 67 & 18.76 & 15.22 & 12.95 & & & & & & & \\
\hline 16 & 27 & 15.83 & -24 & 25 & 13.93 & 20.80 & 16.02 & 13.22 & 68 & 20.23 & 15.90 & 13.26 & 313 & 20.289 & 15.988 & 13.283 & & & \\
\hline 16 & 27 & 15.83 & -24 & 34 & 06.74 & $\geqslant 21.00$ & 19.07 & 17.49 & & & & & 5771 & 20.936 & 19.247 & 17.686 & & & \\
\hline 16 & 27 & 15.90 & -24 & 22 & 53.23 & $\geqslant 21.00$ & 19.73 & 17.94 & & & & & 7906 & 21.006 & 19.460 & 18.198 & & & \\
\hline 16 & 27 & 17.38 & -24 & 32 & 06.97 & $\geqslant 21.00$ & 17.84 & 15.73 & & & & & 1604 & 19.552 & 17.594 & 15.848 & & & \\
\hline 16 & 27 & 17.40 & -24 & 22 & 28.27 & 20.14 & 15.90 & 13.56 & & & & & 341 & 19.672 & 15.605 & 13.430 & & & \\
\hline 16 & 27 & 18.17 & -24 & 25 & 55.52 & $\geqslant 21.00$ & 18.45 & 16.93 & & & & & 3872 & 20.056 & 18.355 & & & & \\
\hline 16 & 27 & 18.33 & -24 & 24 & 25.75 & 16.87 & 13.33 & 11.28 & & & & & 107 & 16.907 & 13.229 & 11.271 & & & \\
\hline 16 & 27 & 19.37 & -24 & 20 & 49.25 & 20.89 & 18.30 & 16.24 & 71 & 21.73 & 18.35 & 16.17 & & & & & & & \\
\hline 16 & 27 & 19.44 & -24 & 26 & 00.82 & $\geqslant 21.00$ & 18.34 & 16.72 & 70 & 21.71 & 18.71 & 16.79 & & & & & & & \\
\hline 16 & 27 & 19.54 & -24 & 26 & 21.28 & $\geqslant 21.00$ & 18.63 & 18.04 & & & & & 5454 & 20.136 & 18.466 & 17.857 & & & \\
\hline 16 & 27 & 19.79 & -24 & 26 & 35.50 & $\geqslant 21.00$ & 19.46 & 18.50 & & & & & 14819 & 20.280 & 19.272 & 18.697 & & & \\
\hline 16 & 26 & 21.07 & -24 & 28 & 28.55 & $\geqslant 21.00$ & 18.79 & 17.30 & & & & & 4823 & 20.559 & 19.048 & 17.432 & & & \\
\hline 16 & 27 & 21.21 & -24 & 37 & 53.59 & $\geqslant 21.00$ & 18.87 & 16.84 & 73 & 22.83 & 19.47 & 17.36 & & & & & & & \\
\hline 16 & 27 & 21.55 & -24 & 21 & 50.85 & 15.59 & 12.63 & 10.98 & & & & & 90 & 15.645 & 12.588 & 11.016 & & & \\
\hline 16 & 27 & 21.66 & -24 & 32 & 17.93 & 20.97 & 18.30 & 16.40 & & & & & 2403 & 18.968 & 17.650 & 16.421 & & & \\
\hline 16 & 27 & 21.99 & -24 & 29 & 38.29 & 19.77 & 15.41 & 12.90 & & & & & 238 & & 15.424 & 12.838 & & & \\
\hline 16 & 27 & 22.46 & -24 & 38 & 37.44 & $\geqslant 21.00$ & 18.63 & 16.96 & 74 & 22.20 & 19.19 & 16.92 & & & & & & & \\
\hline 16 & 27 & 22.90 & -24 & 18 & 26.38 & $\geqslant 21.00$ & 19.26 & 18.03 & & & & & 5757 & 20.668 & 19.698 & 17.982 & & & \\
\hline 16 & 27 & 22.97 & -24 & 22 & 37.01 & $\geqslant 21.00$ & 18.79 & 17.20 & & & & & 3809 & 20.587 & 18.893 & 17.066 & & & \\
\hline 16 & 27 & 23.58 & -24 & 30 & 46.55 & $\geqslant 21.00$ & 19.13 & 17.26 & & & & & 4264 & 19.205 & 18.177 & 17.286 & & & \\
\hline 16 & 27 & 23.59 & -24 & 34 & 44.30 & $\geqslant 21.00$ & 19.38 & 18.03 & & & & & 6745 & 19.838 & 19.049 & 18.329 & & & \\
\hline 16 & 27 & 24.17 & -24 & 25 & 10.86 & $\geqslant 21.00$ & 18.16 & 15.81 & 75 & 22.32 & 18.73 & 15.85 & & & & & & & \\
\hline 16 & 27 & 24.29 & -24 & 20 & 44.72 & $\geqslant 21.00$ & 18.69 & 17.05 & & & & & 5084 & 20.888 & 19.267 & & & & \\
\hline 16 & 27 & 24.37 & -24 & 41 & 48.29 & 18.91 & 15.04 & 12.70 & 76 & 18.78 & 15.01 & 12.72 & 222 & 18.556 & 15.001 & 12.692 & & & \\
\hline 16 & 27 & 24.60 & -24 & 28 & 49.30 & $\geqslant 21.00$ & $\geqslant 21.00$ & 18.50 & & & & & 4077 & 19.920 & 18.673 & & & & \\
\hline
\end{tabular}


Table 4

(Continued)

\begin{tabular}{|c|c|c|c|c|c|c|c|c|c|c|c|c|c|c|c|c|c|c|c|}
\hline \multicolumn{3}{|c|}{ R.A. (2000) } & \multicolumn{3}{|c|}{ Decl. (2000) } & \multicolumn{3}{|c|}{ This Work } & \multicolumn{4}{|c|}{ Alves de Oliveira et al. } & \multicolumn{4}{|c|}{ Marsh et al. } & \multicolumn{3}{|c|}{ Geers et al. } \\
\hline $\mathrm{h}$ & $\mathrm{m}$ & $\mathrm{s}$ & $\circ$ & ' & " & $J$ & $H$ & $K_{s}$ & $\begin{array}{c}\text { Source } \\
\text { No. }\end{array}$ & $J$ & $H$ & $K_{s}$ & $\begin{array}{c}\text { Source } \\
\text { No. }\end{array}$ & $J$ & $H$ & $K_{s}$ & $\begin{array}{c}\text { Source } \\
\text { No. }\end{array}$ & $J$ & $K_{s}$ \\
\hline 16 & 27 & 24.64 & -24 & 34 & 22.04 & $\geqslant 21.00$ & 18.15 & 16.45 & & & & & 2448 & 19.868 & 17.934 & 16.415 & & & \\
\hline 16 & 27 & 24.67 & -24 & 29 & 34.12 & 19.37 & 14.88 & 12.48 & & & & & 195 & 19.268 & 14.836 & 12.474 & & & \\
\hline 16 & 27 & 25.42 & -24 & 25 & 37.51 & $\geqslant 21.00$ & 18.76 & 18.15 & & & & & $4450^{c}$ & 19.573 & 18.335 & 17.709 & & & \\
\hline 16 & 27 & 25.62 & -24 & 35 & 05.79 & $\geqslant 21.00$ & 19.22 & 18.41 & & & & & 7704 & 19.653 & 19.054 & 18.642 & & & \\
\hline 16 & 27 & 25.64 & -24 & 37 & 27.81 & 18.33 & 16.59 & 15.23 & 77 & 18.23 & 16.54 & 15.36 & & & & & & & \\
\hline 16 & 27 & 25.75 & -24 & 29 & 53.59 & $\geqslant 21.00$ & 18.34 & 17.43 & & & & & 4114 & 19.204 & 18.119 & 17.253 & & & \\
\hline 16 & 27 & 25.97 & -24 & 28 & 56.75 & $\geqslant 21.00$ & 19.52 & 17.83 & & & & & 2993 & 20.532 & 18.752 & 17.176 & & & \\
\hline 16 & 27 & 26.18 & -24 & 19 & 23.03 & 16.42 & 14.32 & 12.83 & 78 & 16.35 & 14.36 & 13.04 & 239 & 16.606 & 14.343 & 12.977 & 21 & 16.40 & 12.93 \\
\hline 16 & 27 & 26.57 & -24 & 25 & 54.39 & 12.79 & 12.31 & 11.58 & & & & & 93 & 13.033 & 12.373 & 11.909 & 22 & 13.00 & 11.84 \\
\hline 16 & 27 & 26.62 & -24 & 40 & 45.13 & 18.61 & 14.94 & 12.60 & 79 & 18.49 & 14.89 & 12.54 & & & & & & & \\
\hline 16 & 27 & 27.09 & -24 & 32 & 16.95 & 18.20 & 14.49 & 12.37 & & & & & 183 & 18.354 & 14.465 & 12.384 & & & \\
\hline 16 & 27 & 27.68 & -24 & 38 & 26.95 & $\geqslant 21.00$ & 18.50 & 16.91 & 80 & 21.94 & 18.94 & 16.95 & & & & & & & \\
\hline 16 & 27 & 28.18 & -24 & 31 & 42.23 & 20.85 & 16.14 & 13.80 & & & & & 408 & 20.695 & 16.032 & 13.760 & & & \\
\hline 16 & 27 & 28.71 & -24 & 17 & 06.52 & 18.64 & 14.86 & 12.80 & & & & & 247 & 18.784 & 14.866 & 12.892 & & & \\
\hline 16 & 27 & 29.30 & -24 & 34 & 07.97 & 17.98 & 14.69 & 12.82 & & & & & 232 & 17.831 & 14.644 & 12.828 & & & \\
\hline 16 & 27 & 29.46 & -24 & 39 & 15.95 & 16.37 & 12.52 & $9.95^{\mathrm{b}}$ & & & & & 62 & 17.088 & 12.966 & 10.162 & & & \\
\hline 16 & 27 & 29.52 & -24 & 19 & 44.80 & $\geqslant 21.00$ & 19.70 & 18.63 & & & & & 9096 & 20.968 & 19.852 & 18.424 & & & \\
\hline 16 & 27 & 29.68 & -24 & 29 & 24.75 & $\geqslant 21.00$ & 16.99 & 14.47 & & & & & 681 & 19.824 & 16.859 & 14.504 & & & \\
\hline 16 & 27 & 30.56 & -24 & 38 & 26.43 & $\geqslant 21.00$ & 19.12 & 18.42 & & & & & 6740 & 19.418 & 18.886 & 17.647 & & & \\
\hline 16 & 27 & 30.62 & -24 & 32 & 34.41 & 12.94 & 12.55 & 12.08 & & & & & 101 & 13.022 & 12.453 & 12.213 & & & \\
\hline 16 & 27 & 30.96 & -24 & 20 & 01.74 & $\geqslant 21.00$ & 18.12 & 17.06 & & & & & 3754 & 20.806 & 19.180 & 17.059 & & & \\
\hline 16 & 27 & 31.07 & -24 & 34 & 02.82 & 13.35 & 11.40 & 10.31 & & & & & 59 & 13.431 & 11.327 & 10.344 & & & \\
\hline 16 & 27 & 31.77 & -24 & 31 & 48.20 & $\geqslant 21.00$ & 18.22 & 16.52 & 83 & 21.92 & 18.47 & 16.44 & & & & & & & \\
\hline 16 & 27 & 32.19 & -24 & 29 & 42.79 & 18.67 & 15.08 & 12.95 & 84 & 18.35 & 15.04 & 13.05 & 334 & & 15.088 & 12.995 & 7 & 18.47 & 13.41 \\
\hline 16 & 27 & 32.51 & -24 & 16 & 04.20 & $\geqslant 21.00$ & 18.14 & 16.39 & 85 & 22.42 & 18.81 & 16.57 & & & & & & & \\
\hline 16 & 27 & 32.53 & -24 & 39 & 46.07 & 20.87 & 18.11 & 16.35 & & & & & 2797 & 19.716 & 17.647 & 16.255 & & & \\
\hline 16 & 27 & 32.70 & -24 & 33 & 23.63 & 16.20 & 12.87 & 11.03 & & & & & 132 & 16.126 & 12.685 & 10.848 & & & \\
\hline 16 & 27 & 32.70 & -24 & 22 & 46.48 & 20.81 & 18.17 & 17.20 & & & & & 6419 & 19.441 & 18.436 & 17.425 & & & \\
\hline 16 & 27 & 32.73 & -24 & 32 & 41.83 & 19.00 & 15.21 & 13.04 & & & & & 491 & 19.231 & 15.795 & 13.377 & & & \\
\hline 16 & 27 & 32.96 & -24 & 28 & 11.15 & 20.87 & 17.45 & 15.02 & & & & & 1307 & 19.781 & 17.189 & 14.922 & & & \\
\hline 16 & 27 & 33.55 & -24 & 22 & 49.12 & 20.37 & 18.25 & 17.12 & & & & & 9558 & 19.768 & 19.332 & 17.708 & & & \\
\hline 16 & 27 & 33.67 & -24 & 30 & 50.99 & $\geqslant 21.00$ & 18.31 & 17.24 & & & & & 5003 & 19.691 & 18.954 & 16.895 & & & \\
\hline 16 & 27 & 33.81 & -24 & 22 & 34.33 & 17.84 & 16.76 & 15.69 & & & & & 2870 & 17.725 & 16.491 & 15.672 & & & \\
\hline 16 & 27 & 34.14 & -24 & 33 & 08.37 & $\geqslant 21.00$ & 18.50 & 17.14 & 87 & 22.22 & 18.96 & 17.03 & & & & & & & \\
\hline 16 & 27 & 35.32 & -24 & 39 & 57.61 & 19.50 & 16.59 & 15.08 & & & & & 2391 & 17.625 & 16.288 & 14.858 & & & \\
\hline 16 & 27 & 37.20 & -24 & 34 & 34.12 & $\geqslant 21.00$ & 18.18 & 16.02 & 92 & 21.92 & 18.21 & 15.97 & & & & & & & \\
\hline 16 & 27 & 37.24 & -24 & 25 & 26.43 & $\geqslant 21.00$ & 18.23 & 16.24 & 93 & 22.31 & 18.81 & 16.18 & & & & & & & \\
\hline 16 & 27 & 37.40 & -24 & 17 & 54.78 & 14.08 & 12.82 & 11.80 & & & & & & & & & 23 & 14.15 & 11.95 \\
\hline 16 & 27 & 38.98 & -24 & 40 & 20.53 & 16.54 & 14.20 & 12.47 & 94 & 16.48 & 14.12 & 12.56 & & & & & $8,24^{\mathrm{d}}$ & 16.54 & 12.29 \\
\hline 16 & 27 & 40.13 & -24 & 26 & 36.60 & $\geqslant 21.00$ & 17.30 & 14.15 & 95 & 21.90 & 17.39 & 14.13 & & & & & & & \\
\hline 16 & 27 & 40.95 & -24 & 28 & 59.55 & 14.74 & 13.88 & 13.07 & 96 & 14.60 & 13.76 & 13.19 & & & & & 25 & 14.66 & 13.10 \\
\hline 16 & 27 & 41.84 & -24 & 42 & 34.99 & 20.38 & 17.47 & 15.18 & & & & & & & & & 9 & 21.32 & 14.97 \\
\hline 16 & 27 & 46.39 & -24 & 31 & 41.02 & 13.78 & 12.28 & 11.23 & & & & & & & & & 26 & 13.83 & 11.32 \\
\hline 16 & 27 & 56.76 & -24 & 28 & 16.74 & $\geqslant 21.00$ & 19.47 & 18.22 & & & & & 6249 & 20.940 & 19.558 & 18.104 & & & \\
\hline
\end{tabular}

Notes.

${ }^{a}$ Source 21 of Table 4 of Alves de Oliveira et al. is a 4".5 binary in our images-their coordinates fall between those of the two components, whereas their photometry agrees with our photometry for the fainter component. We list the coordinates and photometry for the primary for completeness.

b Saturated in our data.

${ }^{c}$ This is the planetary mass object confirmed spectroscopically by Marsh et al. (2010a). See the text for discussion.

d Sources 8 and 24 as listed in Geers et al.'s Table 1 have identical entries for their coordinates and photometry, with the exception of the $K_{s}$ photometry, which is listed at 13.36 for Source 8 and as 12.29 for Source 24. We have used the value listed for Source 24, as it agrees better with other determinations.

${ }^{\text {e }}$ Source No. from Table 2 of Geers et al. with spectroscopic follow-up.

and $K_{s}$ values for the spectroscopically confirmed PMO, Object 4450. Our $K_{s}$ photometry agrees with that of Marsh et al. within $0.1 \mathrm{mag}$ for Source 1307, within $0.21 \mathrm{mag}$ for Source 2438, and within 0.02 mag for Source 2403, in agreement with Alves de Oliveira's stated range of magnitude differences for these sources. Our $K_{s}$ value for Object 4450 is $18.15,0.44$ mag fainter than Marsh et al.'s $K_{s}$ value of 17.71, but 1.01 mag brighter than Alves de Oliveira's $K_{s}$ value of 19.14. Alves de Oliveira et al. could not derive an $H$-band value for Object 4450 , due to an image artifact in their data. We derive $H=18.76$ compared with Marsh et al.'s value of $H=18.36$. We did not detect Object 4450 at $J$ band, whereas Marsh et al. derived $J=19.57$ and Alves de Oliveira derived $J=21.32 \pm 0.35$. Based on the large discrepancy between their $K_{S}$-band value and Marsh et al.'s for Object 4450, Alves de Oliveira et al. suggest that this PMO lies as far as the Sco-Cen association and is not 


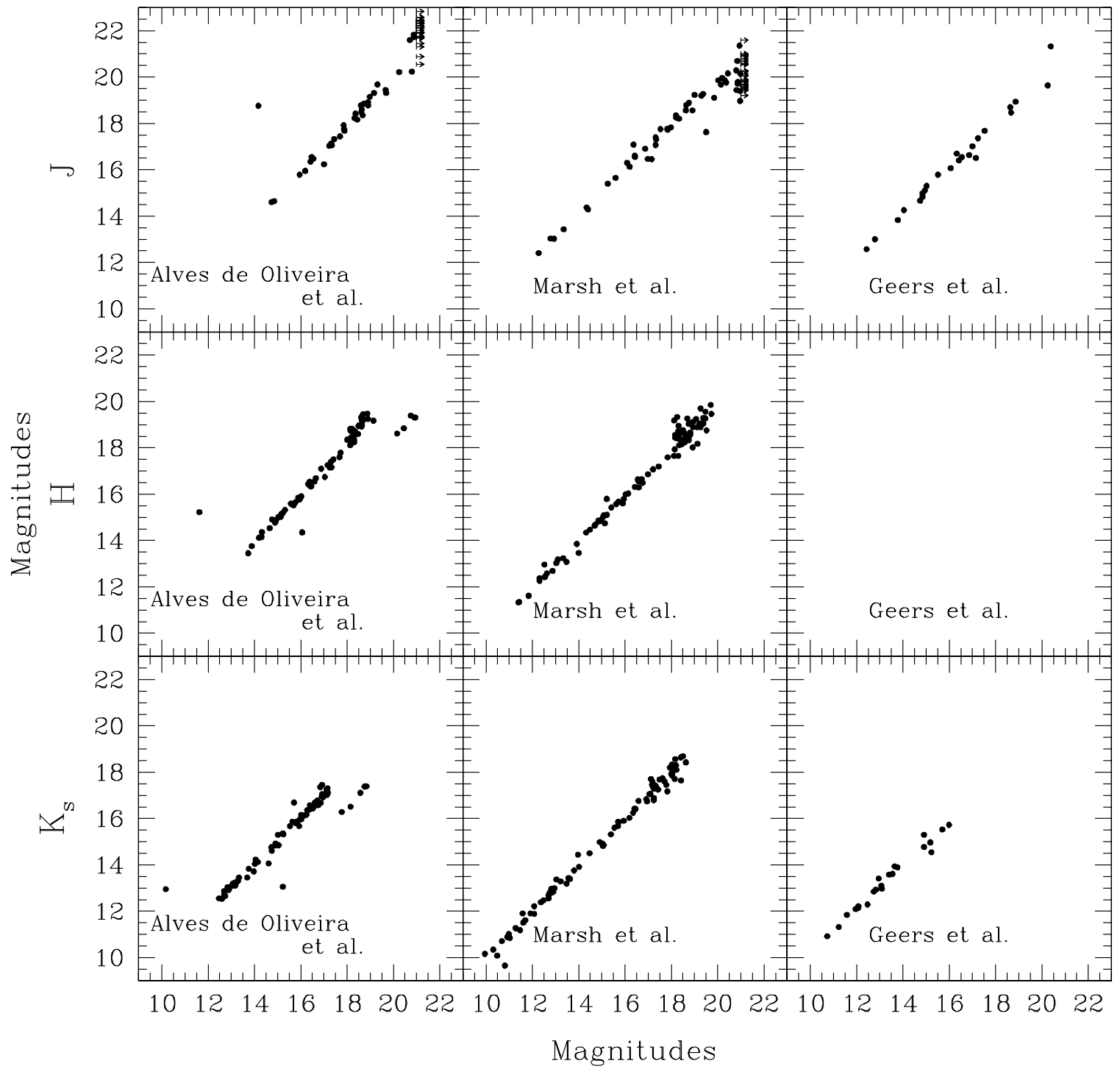

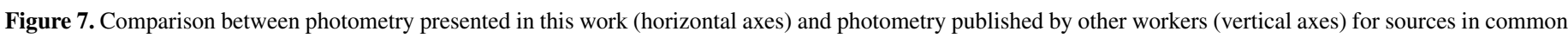

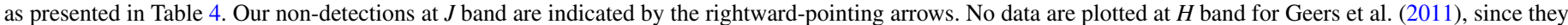
did not obtain $H$-band photometry.

associated with the $\rho$ Oph cloud. Our results do not support this conclusion.

There is a possible scenario that would resolve the issue of such large photometric discrepancies being reported by different workers for the PMO 4450. Note that the observed large differences in photometry between Marsh et al. and Alves de Oliveira et al. occur for 4/15 sources, suggesting the presence of a systematic rather than random measurement error. One possibility is that of extended emission surrounding the objects with discrepant photometry, which we argue to be the case for Object 4450. The pixel size of 2MASS was $2^{\prime \prime}$ (used for the Marsh et al. photometry); the pixel size reported in this work was 0.45 (albeit in 1".0-1".4 seeing), and the pixel size for the Canada-France-Hawaii Telescope (CFHT) WIRCam observations was 0.3 , in excellent seeing, "typically between 0.4 and 0.'5," but always better than 0.'8. Alves de Oliveira used point-spread function (PSF) fitting photometry, which would miss measuring any extended flux and would result in fainter measured magnitudes than would be derived for an extended object from aperture photometry. The hypothesis of an extended source is supported by our measurements falling between the values found by Alves de Oliveira on the one hand and those found by Marsh et al. on the other.

Of the 165 sources listed in Table 1 of Marsh et al. (2010b), photometries for 92 are listed in Table 4. Of the 73 sources not listed in Table 4, 50 are outside of our survey area, 20 are saturated in our data, and 3 are below our detection threshold. The general photometric agreement between the two data sets is satisfactory and is plotted in the middle column of Figure 7.

Geers et al. (2011) list $36^{14}$ "likely substellar members with disks" in $\rho$ Oph in their Table 1, of which 10 have NIR photometry from MOIRCS and 27 have NIR photometry from 2MASS. They list a further three "Probable Low-Mass and Substellar Members of $\rho$ Oph with MOIRCS Spectroscopy Follow-up" in their Table 2. We recover all but four sources, of which three (their Table 1 entries 11, 15, and 27) were outside of our survey limits, and one (their their Table 1, entry 10) which was too faint at $J$ to be detected by our survey and is located in regions of very bad signal to noise in our survey at $H$ and $K$.

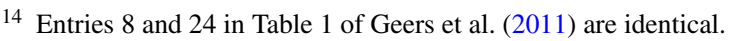


The photometric agreement between our surveys at $J$ and $K_{s}$ (no $H$-band data were acquired by Geers et al. 2011) is quite good, as can be seen from the plots in the right column of Figure 7.

Recently, Geers et al. (2011) have estimated an upper limit for the ratio of low-mass stars $\left(0.1 M_{\odot} \leqslant M \leqslant 1.0 M_{\odot}\right)$ to brown dwarfs $\left(M \leqslant 0.1 M_{\odot}\right)$ in the $\rho$ Ophiuchi cloud to be $\sim 3-7$. An alternative upper limit to the low-mass star:brown dwarf ratio in $\rho$ Oph can be derived using the subsample of "non-excess" sources lying above the main sequence (as plotted in the middle panel of Figure 4).

Only the "non-excess" sources whose SED fits had fluxes within a factor of three of what a photospheric model would predict at the distance of $\rho \mathrm{Oph}$ are considered, in order to exclude YSOs with non-photospheric mid-IR emission that might masquerade as having low-temperature photospheres (see discussion of this point in Section 4.1). Mass estimates for these sources are derived from their best-fit, 1 Myr COND and DUSTY models, yielding 59 objects with masses in the range 0.1 $\leqslant M \leqslant 1.0 M_{\odot}$ and 83 objects with $M \leqslant 0.1 M_{\odot}$, thus yielding a value of $\sim 0.7$ for the low-mass star:brown dwarf ratio. However, unaccounted-for systematic biases (e.g., underestimating the number of higher mass PMS sources) may have entered into this estimate. The point is to illustrate the variation in the possible range of this value in $\rho \mathrm{Oph}$, given the present data. Marsh et al. (2010b) have recently published an estimate of the IMF in the $\rho$ Ophiuchi cloud. Their results show an increase in the number of cloud members progressing from $0.1 M_{\odot}$ to lower masses. Our results, so far, are consistent with theirs. However, it would be premature to derive a definitive IMF across the substellar boundary from the data presented here. Future spectroscopy of our candidate cloud members will allow the construction of an IMF for this region and further refine the value for the low-mass star:brown dwarf ratio in $\rho$ Ophiuchi.

\section{SUMMARY AND CONCLUSIONS}

1. We present a new, deep, $J H K_{s}$ survey of a $920 \operatorname{arcmin}^{2}$ area of the $\rho$ Ophiuchi star-forming cloud, encompassing its highest extinction core to $90 \%$ completeness limits of $J=20.0, H=20.0$, and $K_{s}=18.50$. Our survey is thus sensitive to an object of just $\sim 1.5 M_{\text {Jup }}$ with an age of $1 \mathrm{Myr}$ and photospheric temperature of $\sim 1100 \mathrm{~K}$ at the distance to $\rho$ Oph. This mass sensitivity falls to 2.0, 4.0, and 8.5 $M_{\text {Jup }}$ for $A_{V}=5,10$, and 15 , respectively.

2 . We combine our new, deep, $J H K_{s}$ photometry with midinfrared Spitzer photometry to produce SEDs for a total of 1741 sources within our survey boundaries. These sources are divided into three groupings according to their placement in the $J-H$ versus $H-K_{s}$ color-color diagram into: (1) 830 "excess" sources, those which deredden to the CTTS locus of Meyer et al. (1997); (2) 533 "non-excess" sources, which fall within the main-sequence reddening band; and (3) 378 sources that can be dereddened to the colors of M7-L0 spectral types with a resulting large variation in their deduced extinction values.

3. An improved version of the fitting procedure of Marsh et al. (2010b) is used to fit atmospheric models (COND, DUSTY, NextGen) and single-temperature blackbody spectra to the observed SEDs for an age of 1 Myr and a distance of $124 \mathrm{pc}$, appropriate for the age and distance of the $\rho$ Oph cloud's YSO population.

4. Of the 827 successful SED fits for "excess" sources, 764 are found to lie above the main sequence. Of the 527 successful SED fits for "non-excess" sources, 184 lie above the main sequence. We therefore identify 948 candidate PMS sources, of which 87 (57 "excess" and 30 "nonexcess") are duplicates with sources listed in Wilking et al. (2008).

5. The fact that 184 "non-excess" and 764 "excess" sources are identified as PMS demonstrates the efficacy of this method for identifying the entire PMS population in the surveyed area, unbiased with respect to the presence or absence of disks.

6. Of the 378 sources with complete $J H K_{s}$ and Spitzer SEDs that can be dereddened to the colors of M7-L0 photospheres in the $J-H$ versus $H-K_{s}$ color-color diagram, the fraction above the main sequence varies from $22 \%$ (82 above main sequence/374 successful SED fits), if all such sources are dereddened to M7 colors, to 95\% (357/377), if all such sources are dereddened to L0 colors, and $78 \%$ (294/377) for sources dereddened to colors that are the average between M7 and L0 colors (close to M9, in practice). Follow-up spectroscopy is required to decide what fraction of this sample represents further augmentation of the PMS population of the cloud, and what fraction are reddened background stars.

7. The embedded population of candidate YSOs in the $\rho$ Oph core is increased by a factor of $\sim 4$ by this study, even allowing for contamination of the cluster-member candidate sample by background galaxies or AGNs.

8. Follow-up spectroscopy of the cluster-member candidates opens up the possibility for determination of the IMF in this star-forming cloud throughout the brown dwarf mass range, reaching well into the planetary mass regime.

We thank the AAO staff for their outstanding support in making our observations possible. We also thank Utah Valley University undergraduate physics student Sherene Higley for her assistance in the reduction of the AAO data. M.B., K.H., and C.M. acknowledge the support of NSF Research at Undergraduate Institutions grants AST-1007928, AST-1009776, and AST-1009590, respectively, for support of this research. M.B. gratefully acknowledges NSF grant AST-0206146, which made further contributions to this work possible. Additional support for this work was provided by the National Aeronautics and Space Administration through Chandra Award Number AR1-2005A and AR1-2005B issued by the Chandra X-Ray Observatory Center, which is operated by the Smithsonian Astrophysical Observatory for and on behalf of NASA under Contract NAS8-39073.

\section{REFERENCES}

Alves de Oliveira, C., Moraux, E., Bouvier, J., et al. 2010, A\&A, 515, 75 Andersen, M., Meyer, M. R., Greissl, J., \& Aversa, A. 2008, ApJ, 683, L183 Baraffe, I., Chabrier, G., Barman, T. S., Allard, F., \& Hauschildt, P. H. 2003, A\&A, 402, 701

Barrado, D., Stelzer, B., Morales-Calderón, M., et al. 2011, A\&A, 526, 21 Barsony, M., Ressler, M. E., \& Marsh, K. A. 2005, ApJ, 630, 381

Bessell, M. S., \& Brett, J. M. 1988, PASP, 100, 1134

Bihain, G., Rebolo, R., Zapatero Osorio, M. R., et al. 2009, A\&A, 506, 1169

Burgess, A. S. M., Moraux, E., Bouvier, J., et al. 2009, A\&A, 508, 823 Caballero, J. A., Béjar, V. J. S., Rebolo, R., et al. 2007, A\&A, 470, 903 Chabrier, G., Baraffe, I., Allard, F., \& Hauschildt, P. H. 2000, ApJ, 542, 464 Cohen, J. G., Frogel, J. A., Persson, S. E., \& Elias, J. H. 1981, ApJ, 249, 481

Comerón, F. 2011, A\&A, 531, 33

Geers, V., Scholz, A., Jayawardhana, R., et al. 2011, ApJ, 726, 23

Gutermuth, R. A., Megeath, S. T., Myers, P. C., et al. 2009, ApJS, 184, 18 
Haisch, K. E., Jr., Barsony, M., \& Tinney, C. 2010, ApJ, 719, L90

Haisch, K. E., Jr., Lada, E. A., \& Lada, C. J. 2000, AJ, 120, 1396

Hauschildt, P. H., Allard, F., \& Baron, E. 1999, ApJ, 512, 377

Hennebelle, P., \& Chabrier, G. 2011, in IAU Symp. 270, Computational Star Formation (Cambridge: Cambridge Univ. Press), 159

Kroupa, P. 2011, in IAU Symp. 270, Computational Star Formation (Cambridge: Cambridge Univ. Press), 141

Lada, C. J., \& Adams, F. C. 1992, ApJ, 393, 278

Lodieu, N., Zapatero Osorio, M. R., Rebolo, R., Martín, E. L., \& Hambly, N. C. 2009, A\&A, 505, 1115

Loinard, L., Torres, R. M., Mioduszewski, A., \& Rodriguez, L. F. 2008, ApJ, 675, L29

Lombardi, M., \& Alves, J. 2001, A\&A, 377, 1023

Lombardi, M., Lada, C. J., \& Alves, J. 2008, A\&A, 489, 143

Luhman, K. L. 2004, ApJ, 617, 1216

Luhman, K. L. 2007, ApJS, 173, 104

Luhman, K. L., Allen, P. R., Espaillat, C., Hartmann, L., \& Calvet, L. 2010, ApJS, 186, 111

Luhman, K. L., Mamajek, E. E., Allen, P. R., \& Cruz, K. L. 2009a, ApJ, 703, 399

Luhman, K. L., Mamajek, E. E., Allen, P. R., Muench, A. A., \& Finkbeiner, D. P. 2009b, ApJ, 691, 1265

Luhman, K. L., \& Rieke, G. H. 1999, ApJ, 525, 440

Luhman, K. L., Stauffer, J. R., Muench, A. A., et al. 2003, ApJ, 593, 1093

Luhman, K. L., Whitney, B. A., Meade, M. R., et al. 2006, ApJ, 647, 1180

Marsh, K. A., Kirkpatrick, J. D., \& Plavchan, P. 2010a, ApJ, 709, L158

Marsh, K. A., Plavchan, P., Kirkpatrick, J. D., et al. 2010b, ApJ, 719, 550

Mayne, N. J., \& Harries, T. J. 2010, MNRAS, 409, 1307

McKee, C., \& Ostriker, E. 2007, ARA\&A, 45, 565

Meyer, M. R., Calvet, N., \& Hillenbrand, L. A. 1997, AJ, 114, 288
Monin, J.-L., Guieu, S., Pinte, C., et al. 2010, A\&A, 515, 91

Muench, A. A., Lada, E. A., \& Alves, J. 2002, ApJ, 573, 366

Muench, A. A., Lada, E. A., Lada, C. J., et al. 2003, AJ, 125, 2029

Mužić, K., Scholz, A., Geers, V., Fissel, L., \& Jayawardhana, R. 2011, ApJ, 732,86

Persson, S. E., Murphy, D. C., Krzeminski, W., Roth, M., \& Rieke, M. J. 1998, AJ, 116, 2475

Pillitteri, I., Sciortino, S., Flaccomio, E., et al. 2010, A\&A, 519, 34

Prato, L., Greene, T. P., \& Simon, M. 2003, ApJ, 584, 853

Preibisch, T., Stanke, T., \& Zinnecker, H. 2003, A\&A, 409, 147

Rebull, L. M., Padgett, D. L., McCabe, C.-E., et al. 2010, ApJS, 186, 259

Retzlaff, J., Rosati, P., Dickison, M., et al. 2010, A\&A, 511, 50

Riaz, B., \& Martín, E. L. 2011, A\&A, 525A, 10

Ridge, N., Di Francesco, J., Kirk, H., et al. 2006, AJ, 131, 2921

Robberto, M., Soderblom, D. R., Scandariato, G., et al. 2010, AJ, 139, 950

Scholz, A., Geers, V., Jayawardhana, R., et al. 2009, ApJ, 702, 805

Slesnick, C. L., Hillenbrand, L. A., \& Carpenter, J. M. 2004, ApJ, 610, 1045

Stephens, D. C., \& Leggett, S. K. 2004, PASP, 116, 9

Stetson, P. 1987, PASP, 99, 191

Terebey, S., van Buren, D., Padgett, D. L., Hancock, T., \& Brundage, M. 1998, ApJ, 507, L71

Todorov, K., Luhman, K. L., \& McLeod, K. K. 2010, ApJ, 714, L84

Weights, D. J., Lucas, P., Roche, P. F., Pinfield, D. J., \& Riddick, F. 2009, MNRAS 817

Wilking, B. A., Gagné, M., \& Allen, L. E. 2008, in Handbook of Star Forming Regions, Vol. II, The Southern Sky, ed. B. Reipurth, ASP Monograph Publications, Vol. 5 (San Francisco, CA: ASP), 351

Wilking, B. A., Meyer, M. R., Robinson, J. G., \& Greene, T. P. 2005, AJ, 130, 1733

Winston, E., Megeath, S. T., Wolk, S. J., et al. 2010, AJ, 140, 266 